\title{
Resonant and off-resonant transients in electromagnetically induced transparency: turn-on and turn-off dynamics
}

\author{
Andrew D. Greentree, T. B. Smith, S. R. de Echaniz, and A. V. Durrant \\ Quantum Processes Group, Department of Physics and Astronomy, \\ The Open University, Walton Hall, Milton Keynes, MKr 6AA, UK. \\ J. P. Marangos and D. M. Segal \\ Laser Optics and Spectroscopy Group, Blackett Laboratory, \\ Imperial College of Science Technology and Medicine, Prince Consort Road, London SW7 2BW, UK. \\ J. A. Vaccaro \\ Department of Physics and Astronomy, University of Hertfordshire, College Lane, Hatfield, AL10 9AB, UK.
}

\begin{abstract}
This paper presents a wide-ranging theoretical and experimental study of non-adiabatic transient phenomena in a $\Lambda$ EIT system when a strong coupling field is rapidly switched on or off. The theoretical treatment uses a Laplace transform approach to solve the time-dependent density matrix equation. The experiments are carried out in $\mathrm{Rb}^{87} \mathrm{MOT}$. The results show transient probe gain in parameter regions not previously studied, and provide insight into the transition dynamics between bare and dressed states.
\end{abstract}

PACS numbers: 42.50.Md, 42.50.Gy, 42.50.Hz, 32.80.Pj

\section{INTRODUCTION}

There is now a huge interest in the novel optical properties of coherently-prepared atomic media, especially electromagnetically induced transparency (EIT) where a resonant coupling field (or pump field) coherently prepares an atomic sample to allow dissipation-free propagation of a weak probe field accompanied by strong dispersion. There have been applications of EIT to enhanced non-linear optical processes [1], laser cooling [2], quantum non-demolition measurements [3] and gain without inversion [4]. EIT is also the mechanism underlying the recent experiments in ultra-slow [5] and ultra-fast group velocities [6] and is potentially of use for storage and retrieval of quantum information using robust ground state coherences [7]. There is also a continuing interest in exploiting the strong optical non-linearities for the control of light by light at the single photon level [8]. A review of EIT and its applications can be found in ref [9].

The study of transient excitation of three level systems is a mature field, but there is still a need for experimental verifications of theoretical work. An early theoretical paper by Berman and Salomaa [10] compared the dressedatom and bare-atom pictures, and considered transients after probe turn-on. Related dressed-atom transients for two level atoms are presented theoretically by $\mathrm{Lu}$ and Berman 11]. Theoretical three-level transient studies considering intial conditions have also appeared in $\mathrm{Lu}$ et al. [12]. Harris and Luo [13] studied transient EIT in the context of the energy required for the preparation of EIT. Li and Xiao [14 investigated the time required for the onset of EIT and Zhu considered the conditions required for observing inversionless gain in the transient regime for the $\mathrm{V}$ 15] and $\Lambda$ 16 schemes.

Experimental work looking at dressed state transients includes phase shifting measurements in a two level system [17], fluorescence measurements on a three level $\Lambda$ system 18] and pump-probe experiments on a three level ladder system 19]. Transient gain was first observed experimentally in a sodium sample in a $\Lambda$ configuration by Fry et al. 20]. This is also the only previous experiment we know of which analyzed transient dynamics associated with the turn-off of the coupling field. RF experiments on the $\mathrm{N}-\mathrm{V}$ centre of diamond appear in [21]. In our earlier work, we have demonstrated transient EIT 22] and Rabi oscillations and gain without inversion 23] in a cold rubidium $\Lambda$ system after rapidly switching on a resonant coupling field.

The work presented here differs from all previous studies in several ways. It extends previous work in transient EIT by studying turn-on and turn-off transients, and the region of parameter space investigated is much broader than previously studied. This is the only work we know of which studies turn-off transients for off-resonant probe and coupling fields, inversionless gain away from resonance and transient dressed state interference away from either bare or dressed state resonance. Furthermore we present new analytical results to describe our experimental transients, derived using the Laplace transform method, so extending the two level approach presented in [24]. Our theoretical results also generalize those of $\mathrm{Li}$ and Xiao [14] for mutual resonance to include two-photon dephasing and arbitrary initial ground state populations, and we predict a new frequency of oscillation in the probe transient response. We also present an analytical solution, to first order in the probe strength, for the probe absorption when the coupling field is non-adiabatically turned off for arbitrary detunings of coupling and probe fields. Our analysis is performed explicitly in the bare state basis. Our results provide insight into how atomic systems become dressed by intense laser fields. 
A natural classification of EIT response with switched fields is based on the relative time scales involved. In the adiabatic regime the switching is assumed to occur sufficiently slowly that the system evolves smoothly from one steady state to another. For EIT systems where the Rabi frequency is comparable to the atomic lifetime, the switching is adiabatic when it occurs on a time scale that is long compared with the relevant optical pumping times. For example, steady state $\Lambda$-type EIT with resonant fields is characterized by the ground state populations being in the non-absorbing superposition of the two ground states. If the intensity ratio or relative phase of the two optical fields changes, then the composition of the non-absorbing superposition state changes, and so the populations have to be pumped into the new nonabsorbing state to maintain transparency. Thus the adiabatic condition requires the switching of the optical fields to be slow compared with the time taken for optical pumping from the absorbing state to the non-absorbing state. This is the regime used in the recent studies of optical information storage and retrieval [7], the single photon switch [25] and its classical precursor [26].

There is another adiabatic regime in pulsed systems where evolution occurs between ground states without spontaneous dissipation. In the work by Harris and Luo [13] on the conditions for preparing EIT, this was achieved by using extremely large Rabi frequencies so that all system evolution to the dark state was achieved on timescales much shorter than the spontaneous emission time. Not requiring such extreme Rabi frequencies, adiabatic switching using the well-known STIRAP schemes [27] decouples the evolution from the spontaneous emission, and so adiabatic evolution occurs on timescales independent of spontaneous emission time. The picosecond pulse experiment of Nottleman et al. 28] is also effectively in the adiabatic regime. In their experiment the relative phase of the two Zeeman ground states oscillates by RF Lamor precession in a magnetic field, and the probe pulses are timed to arrive at the particular times in the RF cycle when the ground states are in the non-absorbing state. We are not concerned with such regimes in this work.

In this paper we are concerned with the non-adiabatic regime, where the switching time is very fast on the scale of optical pumping times, and all other relevant time scales, with the coupling field strength comparable to the spontaneous emission rate. The theoretical studies by $\mathrm{Li}$ and Xiao [14] and Zhu [16] assume (as we do) instantaneous switching and are therefore in the non-adiabatic regime, as are the three experimental observations in $\Lambda$ systems [20, 22, 23] where the coupling field was rapidly switched using a Pockels cell. These studies were restricted to special cases of resonant fields and some specific detunings.

There has also been interest in a further non-adiabatic time regime where effects are studied on timescales comparable with the transition period, see for example 29] where the Rabi frequency of the coupling field was com- (a)
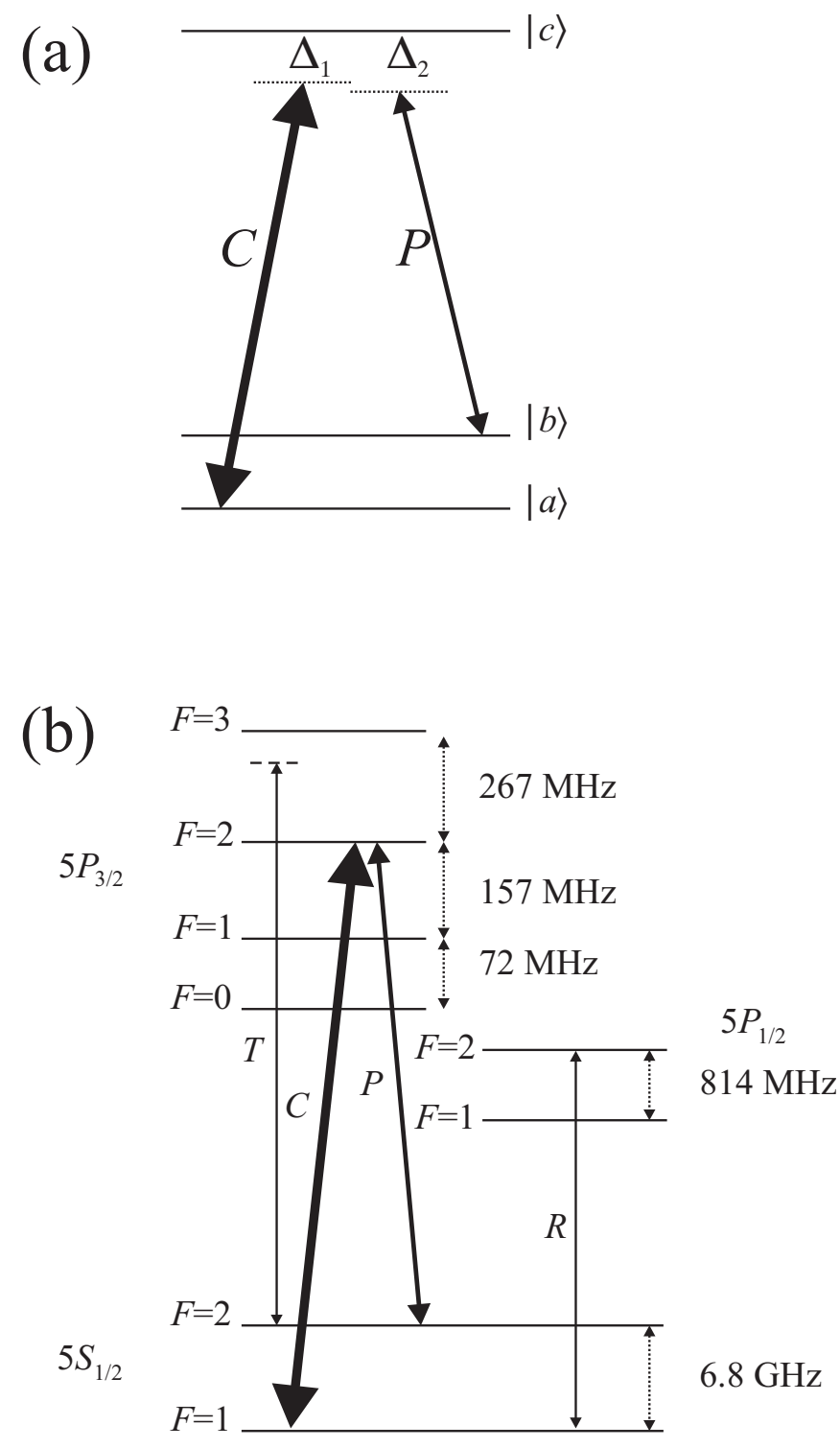

FIG. 1: (a) Model energy level diagram. The atomic energy levels are labelled $|a\rangle,|b\rangle$, and $|c\rangle$ in order of increasing energy. The $|a\rangle-|c\rangle$ transition is driven by a strong coupling field, $C$, with detuning, $\Delta_{1}$ and Rabi frequency, $\Omega_{1}(t)$. The $|b\rangle-|c\rangle$ transition is monitored by a weak probe , $P$, of detuning, $\Delta_{2}$ and Rabi frequency $\Omega_{2}<\Gamma$. (b) Experimental realization in ${ }^{87} \mathrm{Rb}$ MOT. The trapping fields $T$ are detuned from the $5 S_{1 / 2} F=1$ to $5 P_{3 / 2} F=3$ transition by $\Delta_{T}=-13 \mathrm{MHz}$ and a repumper, $R$, is applied resonantly with the $5 S_{1 / 2} F=1$ to $5 P_{1 / 2} F=2$ transition. The coupling field is applied to the $5 S_{1 / 2} F=1$ to $5 P_{3 / 2} F=2$ transition and is switched nonadiabatically using a Pockels cell. The probe is applied to the $5 S_{1 / 2} F=2$ to $5 P_{3 / 2} F=2$ transition. Both coupling and probe fields were held at constant detunings for data collection.

parable to the transition frequency in a Galium-Arsenide semiconductor, and in the N-V centre of diamond [30] where RF transitions at the Rabi frequency were further driven by intense fields. Such regimes are quite different 
from those considered here as they go beyond conventional Bloch analysis of the atomic evolution.

The organization of this paper is as follows. Section [I] outlines the Laplace transform method for calculating transient evolution of an ideal $\Lambda$ system, presents some simple analytical results for coupling field turn-on and turn-off, and gives an overview of the general predictions for arbitrary detunings of both fields. Section III describes the experimental realization of a $\Lambda$ system in laser-cooled rubidium and presents the experimental details. The results of the turn-on and turn-off experiments are presented in Section [V] together with the theoretical predictions. The results are discussed in Section $\mathrm{V}$.

\section{TRANSIENTS IN A $\Lambda$ SYSTEM USING LAPLACE TRANSFORMS}

We consider an ideal closed $\Lambda$ system of three nondegenerate levels excited by monochromatic laser beams, as shown in FIG. 11(a). The sample is assumed to be optically thin and so propagation effects are not considered. The atomic energy levels are labelled in order of increasing energy $|a\rangle,|b\rangle$ and $|c\rangle$. The $|a\rangle-|c\rangle(|b\rangle-|c\rangle)$ transition is coupled by an optical field with frequency $\omega_{1}\left(\omega_{2}\right)$ and detuning $\Delta_{1}=\omega_{1}-\omega_{c a}\left(\Delta_{2}=\omega_{2}-\omega_{c b}\right)$. The transition frequencies are defined $\omega_{\beta \alpha}=\omega_{\beta}-\omega_{\alpha}$, $\alpha, \beta=a, b, c$ and in our system $\omega_{b a} \ll \omega_{c a}, \omega_{c b}$. For convenience we also define $\Delta_{21}=\Delta_{2}-\Delta_{1}$. The state $|c\rangle$ is an excited state which decays by spontaneous emission to the ground (or metastable) states $|a\rangle$ and $|b\rangle$ at rates $\Gamma_{c a}$ and $\Gamma_{c b}$ respectively. There is assumed to be decay of the (two-photon) coherence on the $|a\rangle-|b\rangle$ transition at a rate $\Gamma_{b a}$, but no incoherent coupling between these two states. The Rabi frequency of field $1(2)$ is $\Omega_{1}=\mathbf{d}_{c a} \cdot \mathbf{E}_{1}$ $\left(\Omega_{2}=\mathbf{d}_{c b} \cdot \mathbf{E}_{2}\right)$ where $\mathbf{d}_{\alpha \beta}$ is the electric-dipole moment of the $|\alpha\rangle-|\beta\rangle$ transition, $\mathbf{E}_{j}$ is the electric vector of optical field $j$, and we have chosen units such that $\hbar=1$ so that energies are measured in units of frequency. Optical field 1 is the pump or coupling field $(C)$ and field 2 is the probe $(P)$. For the purposes of this work we usually assume that the probe is weak, i.e. $\Omega_{2}<<\Gamma_{c b}$ although the density matrix equations are presented without this assumption.

In the bare state basis, the density matrix equations of motion for the system in the rotating frame are

$$
\begin{aligned}
\dot{\rho}_{a a} & =\Gamma_{c a} \rho_{c c}+i \frac{\Omega_{1}(t)}{2}\left(\rho_{c a}-\rho_{a c}\right), \\
\dot{\rho}_{a b} & =\left(i \Delta_{21}-\Gamma_{b a}\right) \rho_{a b}+i\left[\frac{\Omega_{1}(t)}{2} \rho_{c b}-\rho_{a c} \frac{\Omega_{2}}{2}\right], \\
\dot{\rho}_{a c} & =\left(-i \Delta_{1}-\frac{\Gamma_{c a}+\Gamma_{c b}}{2}\right) \rho_{a c}+i\left[\left(\rho_{c c}-\rho_{a a}\right) \frac{\Omega_{1}(t)}{2}-\rho_{a b} \frac{\Omega_{2}}{2}\right], \\
\dot{\rho}_{b b} & =\Gamma_{c b} \rho_{c c}+i \frac{\Omega_{2}}{2}\left(\rho_{c b}-\rho_{b c}\right), \\
\dot{\rho}_{b c} & =\left(-i \Delta_{2}-\frac{\Gamma_{c a}+\Gamma_{c b}}{2}\right) \rho_{b c}+i\left[-\rho_{b a} \frac{\Omega_{1}(t)}{2}+\left(\rho_{c c}-\rho_{b b}\right) \frac{\Omega_{2}}{2}\right], \\
\dot{\rho}_{c c} & =-\left(\Gamma_{c a}+\Gamma_{c b}\right) \rho_{c c}+i\left[\frac{\Omega_{1}(t)}{2}\left(\rho_{a c}-\rho_{c a}\right)+\frac{\Omega_{2}}{2}\left(\rho_{b c}-\rho_{c b}\right)\right], \\
\rho_{\alpha \beta} & =\rho_{\beta \alpha}^{*} \\
1 & =\rho_{a a}+\rho_{b b}+\rho_{c c} .
\end{aligned}
$$

The method for switching between bare and dressed bases is given in 10]. Because we measure probe absorption, which is proportional to $\operatorname{Im}\left[\rho_{b c}(t)\right]$, we concentrate on this component of the density matrix.

The standard approach to solving Eqs. (1) is to numerically integrate them (see for example 16]). This method has the advantage of conceptual ease and has been used to generate some of the theoretical turn-on results presented in this work. However this approach has the deficiencies that it is not analytic and therefore gives minimal insight into the underlying dynamics of the problem, and that it can suffer cumulative nu- merical errors. Accordingly, we have also considered a Laplace transform solution which can avoid these difficulties. The Laplace method (in appropriate limits) is more amenable to analysis and in this work we present what we believe to be several new analytical results, especially the turn-off equations. We find an extra frequency of oscillation when the system responds to turning on the coupling field, and suggest the regime where this might be observable. Even when the Laplace method cannot easily produce simple explicit formulae, Eqs. (11) are linear so that any solution depends only linearly on the initial conditions, and so avoids the problem of cumulative 
numerical errors, making the solutions more robust.

Any solution to Eqs. (11) has the form

$$
\rho_{\alpha \beta}(t)=\sum_{l} a_{l} e^{b_{l} t} .
$$

Our goal therefore is to determine coefficients $a_{l}$ and $b_{l}$. The difficulty lies in the fact that the coefficients depend on some or all of the system parameters, including $\Omega_{1}$ and $\Omega_{2}$.

Defining the Laplace transform of $\rho_{\alpha \beta}(t)$ to be $r_{\alpha \beta}(p)$ we have 31]

$$
r_{\alpha \beta}(p)=\int_{0}^{\infty} d t e^{-p t} \rho_{\alpha \beta}(t)
$$

and the transform of $\dot{\rho}_{\alpha \beta}(t)$ is $p r_{\alpha \beta}(p)-\rho_{\alpha \beta}^{0}$ where $\rho_{\alpha \beta}^{0}=\rho_{\alpha \beta}(0)$. Then Eqs. (11) generate nine coupled algebraic equations for the $r_{\alpha \beta}(p)$. By manipulating these equations, one finds four closed equations for $r_{a c}$, $r_{c a}, r_{b c}$, and $r_{c b}$. One of them is

$$
\begin{aligned}
& r_{a c}(p)\{p+\left.i \Delta_{1}+\frac{\Gamma_{c a}+\Gamma_{c b}}{2}+\frac{1}{p} \frac{\Omega_{1}^{2}}{4}[1-s(p)]+\frac{\left(\Omega_{2} / 2\right)^{2}}{p-i \Delta_{21}+\Gamma_{b a}}\right\} \\
&+r_{c a}(p) \frac{1}{p} \frac{\Omega_{1}^{2}}{4}[s(p)-1]-r_{b c}(p) \frac{1}{p} \frac{\Omega_{1} \Omega_{2}}{4} s(p)+r_{c b}(p) \frac{1}{p} \frac{\Omega_{1} \Omega_{2}}{4}\left[s(p)-\frac{p}{p-i \Delta_{21}+\Gamma_{b a}}\right] \\
& \quad=\rho_{a c}^{0}-\frac{i}{p} \frac{\Omega_{1}}{2}\left[\rho_{a a}^{0}+\rho_{c c}^{0} s(p)\right]-i \frac{\Omega_{2}}{2} \frac{\rho_{a b}^{0}}{p-i \Delta_{21}+\Gamma_{b a}},
\end{aligned}
$$

with

$$
s(p)=\frac{\Gamma_{c a}-p}{p+\left(\Gamma_{c a}+\Gamma_{c b}\right)} .
$$

The remaining three equations can be obtained from Eq. (3) by (i) taking the complex conjugate of Eq. (3), treating $p$ as real, (ii) interchanging all labels $a$ and $b$, and 1 and 2, in Eq. (3) and (iii) taking the complex conjugate of the latter 'interchanged' equation. Keeping $p$ real in these procedures is merely a formal device. Once the equations are written by the scheme given, $p$ may take on complex values, as it must when one effects the Laplace inversion to get $\rho_{\alpha \beta}(t)$.

The four coupled equations allow for arbitrary initial values $\rho_{\alpha \beta}^{0}$. Finally when $r_{a c}, r_{b c}, r_{c a}$ and $r_{c b}$ are known, one has

$$
\begin{aligned}
& r_{a b}(p)=\frac{\rho_{a b}^{0}+i \frac{\Omega_{1}}{2} r_{c b}(p)-i \frac{\Omega_{2}}{2} r_{a c}(p)}{p-i \Delta_{21}+\Gamma_{b a}}, \\
& r_{b a}(p)=\frac{\rho_{b a}^{0}+i \frac{\Omega_{2}}{2} r_{c a}(p)-i \frac{\Omega_{1}}{2} r_{b c}(p)}{p+i \Delta_{21}+\Gamma_{b a}},
\end{aligned}
$$

and the diagonal elements are

$$
\begin{aligned}
r_{a a}(p)= & \frac{1}{p}\left[\rho_{a a}^{0}+\Gamma_{c a} r_{c c}(p)\right] \\
& +i \frac{1}{p} \frac{\Omega_{1}}{2}\left[r_{c a}(p)-r_{a c}(p)\right], \\
r_{b b}(p)= & \frac{1}{p}\left[\rho_{b b}^{0}+\Gamma_{c b} r_{c c}(p)\right] \\
& +i \frac{1}{p} \frac{\Omega_{2}}{2}\left[r_{c b}(p)-r_{b c}(p)\right],
\end{aligned}
$$

where

$$
\begin{aligned}
r_{c c}(p)= & \frac{\rho_{c c}^{0}+i \frac{\Omega_{1}}{2}\left[r_{a c}(p)-r_{c a}(p)\right]}{p+\Gamma_{c a}+\Gamma_{c b}} \\
& +\frac{i \frac{\Omega_{2}}{2}\left[r_{b c}(p)-r_{c b}(p)\right]}{p+\Gamma_{c a}+\Gamma_{c b}} .
\end{aligned}
$$

Having solved the equations for the $r_{\alpha \beta}(p)$, one generates the $\rho_{\alpha \beta}(t)$ by Laplace inversion, namely

$$
\rho_{\alpha \beta}(t)=\frac{1}{2 \pi i} \int_{\mathrm{C}} d p e^{p t} r_{\alpha \beta}(p),
$$

where the contour $\mathrm{C}$ in the complex $p$-plane runs vertically from $\sigma-i \infty$ to $\sigma+i \infty$ and $\sigma$ is real and chosen sufficiently large so that $\mathrm{C}$ runs to the right of all poles of $r_{\alpha \beta}(p)$.

Equations (3) to (8) yield solutions of the form

$$
r_{\alpha \beta}(p)=\frac{\mathcal{C}_{\alpha \beta}(p)}{\mathcal{P}_{\alpha \beta}(p)},
$$

where the $\mathcal{C}_{\alpha \beta}(p)$ are polynomials which depend on the parameters of the problem, and in particular, linearly upon one or more of the initial values $\rho_{\alpha \beta}^{0}(0)$. The $\mathcal{P}_{\alpha \beta}(p)$ are polynomials too, but do not depend on the initial values. Generally the zeros of $\mathcal{P}_{\alpha \beta}(p)$ in the complex plane are distinct, so that $r_{\alpha \beta}(p)$ may be expanded as partial fractions

$$
r_{\alpha \beta}(p)=\sum_{l} \frac{\mathcal{R}_{\alpha \beta, l}(p)}{p-P_{\alpha \beta, l}}
$$


where $P_{\alpha \beta, l}$ is the $l^{\text {th }}$ root of the equation $\mathcal{P}_{\alpha \beta}(p)=0$ and the $\mathcal{R}_{\alpha \beta, l}(p)$ are determined using standard algebra. Using this in the contour integral, Eq. (9) gives

$$
\rho_{\alpha \beta}(t)=\sum_{l} \mathcal{R}_{\alpha \beta, l}\left(P_{\alpha \beta, l}\right) e^{P_{\alpha \beta, l} t} .
$$

Although the principles of this approach are simple, the difficulty lies in finding the zeros of the $\mathcal{P}_{\alpha \beta}(p)$, which may have to be done numerically or by some approximation. Most roots $P_{\alpha \beta, l}$ give rise to damped oscillatory terms or to simple damping, but should there be a pole at the origin, its contribution does not decay and represents a stationary state. Such a long-time limit, should it exist, is

$$
\lim _{t \rightarrow \infty} \rho_{\alpha \beta}(t)=\mathcal{R}_{\alpha \beta}(0)=\lim _{p \rightarrow 0}\left[\frac{p \mathcal{C}_{\alpha \beta}(p)}{\mathcal{P}_{\alpha \beta}(p)}\right] .
$$

To facilitate matters, at very little cost, we shall henceforth take $\Gamma_{c a}=\Gamma_{c b}=\Gamma$.

We now present a simple illustrative example of the Laplace transform method, showing explicit expressions for optical pumping due to the probe only. This is followed by application of the method to the transients following turn-on and turn-off of the coupling field. The latter solutions are provided to first order in the probe intensity. For simplicity the turn-on expression is only given for resonant fields, however the turn-off result is presented for arbitrary detunings. This section ends with a graphical overview and discussion of the general turn-on and turn-off dynamics.

\section{A. Optical pumping by a probe}

As an illustration, we consider optical pumping by the probe in the absence of a coupling field $\left(\Omega_{1}=0\right)$. This simple illustrative example encapsulates the Laplace method. A Schrödinger equation approach to this familiar problem appears in [32]. We suppose that when the probe is turned on, at time zero, all three states may be populated but that there are no coherences, i.e. $\rho_{\alpha \beta}^{0}$ may be non-zero for $\alpha=\beta$ only. Then, when Eq. (3) and the associated interchanged equations are solved, one finds that only $\rho_{b c}, \rho_{c b}, \rho_{a a}, \rho_{b b}$ and $\rho_{c c}\left(=1-\rho_{a a}-\rho_{b b}\right)$ are in general non-zero. Use of Mathematica 4.1 [33] to do the algebra, yields

$$
\begin{aligned}
& r_{b c}(p)=-\frac{\Omega_{2}}{2} \frac{\left[\Gamma\left(1-\rho_{a a}^{0}+\rho_{b b}^{0}\right)+p\left(2 \rho_{b b}^{0}+\rho_{a a}^{0}-1\right)\right]\left[i(p+\Gamma)+\Delta_{2}\right]}{D(p)}, \\
& r_{a a}(p)=\frac{p\left[\Gamma+\rho_{a a}^{0}(\Gamma+p)-\rho_{b b}^{0} \Gamma\right]\left[\Delta_{2}^{2}+(p+\Gamma)^{2}\right]+2(p+\Gamma)\left(\Gamma+2 \rho_{a a}^{0} p\right)\left(\Omega_{2} / 2\right)^{2}}{p D(p)}, \\
& r_{b b}(p)=\frac{\left[\Gamma\left(1-\rho_{a a}^{0}+\rho_{b b}^{0}\right)+\rho_{b b}^{0} p\right]\left[\Delta_{2}^{2}+(p+\Gamma)^{2}\right]+2(p+\Gamma)\left(\rho_{a a}^{0}-1\right)\left(\Omega_{2} / 2\right)^{2}}{D(p)},
\end{aligned}
$$

where

$$
D(p)=p(p+2 \Gamma)\left[\Delta_{2}^{2}+(p+\Gamma)^{2}\right]+2(p+\Gamma)(2 p+\Gamma)\left(\Omega_{2} / 2\right)^{2} .
$$

Furthermore, $r_{c b}(p)$ is obtained by complex conjugation of $r_{b c}$, holding $p$ real, and then allowing $p$ to be complex. Then the $\rho_{\alpha \beta}(t)$ follow by Eq. (9). Applying the limit in Eq. (12) to these quantities shows that all $\rho_{\alpha \beta}(t)$ except $\rho_{a a}$ approach zero as $t \rightarrow \infty$, and $\rho_{a a}(t) \rightarrow 1$ in that limit. This simply means that even a weak probe field will pump the system into state $|a\rangle$ after a long time, the usual result. A close look, however, shows that for a weak probe field $\left(\Omega_{2}<\Gamma\right)$ there are three important time periods. Since $D(p)$ is a fourth degree polynomial in $p$, it has four roots. The real parts of three of them are of order $-\Gamma$ or $-2 \Gamma$ but the fourth root is much smaller. To see this, we set $D(p)=0$ and assume that terms of order $p^{2}$ and higher are small. The fourth root $p_{4}$ is then approximated by

$$
p_{4} \approx-\frac{\left(\Omega_{2} / 2\right)^{2}}{\Delta_{2}^{2}+\Gamma^{2}} \Gamma .
$$

Thus, there is an initial period of damped oscillations in $\rho_{\alpha \beta}(t)$, which die out in times of order $\Gamma^{-1}$ or $(2 \Gamma)^{-1}$. Optical coherences form in an intermediate period after the initial dampening, and then a longer period obtains when $\rho_{b c}(t), \rho_{b b}(t)$ and $\rho_{c c}(t)$ very slowly damp to zero and $\rho_{a a}(t)$ slowly approaches unity. This latter time period derives from the probe optical pumping. For small $\Omega_{2}$, the intermediate period is long and lies between 
$1 /(2 \Gamma)$ and $1 / p_{4}$. For this epoch, Eqs. (13), (14) and (15) are well approximated by expanding them to first order in $\Omega_{2}$. When this is done and the limit 12 is taken, one finds

$$
\begin{aligned}
& \rho_{b c}(t) \rightsquigarrow-\frac{\Omega_{2}}{4} \frac{1-\rho_{a a}^{0}+\rho_{b b}^{0}}{\Delta_{2}-i \Gamma}, \\
& \rho_{a a}(t) \rightsquigarrow \frac{1+\rho_{a a}^{0}-\rho_{b b}^{0}}{2}, \\
& \rho_{b b}(t) \rightsquigarrow \frac{1-\rho_{a a}^{0}+\rho_{b b}^{0}}{2} .
\end{aligned}
$$

More generally, although the equation $D(p)=0$ is of fourth order and exactly solvable, numerical values for $\rho_{\alpha \beta}(t)$ are perhaps best achieved by numerically finding the roots $p_{1}$ to $p_{4}$.

\section{B. Turn-on transient with resonant fields}

We now shift the time origin to the instant of turn-on of the coupling field and use Eqs. (3) to (8) to find $\operatorname{Im}\left[\rho_{b c}(t)\right]$ after $t=0$. It is assumed that the probe has been on for some time in order to establish values for $\rho_{a a}^{0}, \rho_{b b}^{0}$ and $\rho_{b c}^{0}=\left(\rho_{c b}^{0}\right)^{*}$. From the previous discussion we know that in our case, these will be the only non-zero values of $\rho_{\alpha \beta}^{0}$. The probe is assumed weak, $\Omega_{2} \ll \Gamma$; therefore we treat the problem to first order in the probe intensity. This corresponds to the experimental situation to be described in Sections III and IV. For simplicity we here assume $\Gamma_{b a}=0$ and resonant excitation, i.e. $\Delta_{1}=\Delta_{2}=0$. We then find, [33], that the Laplace transform of $\operatorname{Im}\left[\rho_{b c}(t)\right]$ (to first order in $\Omega_{2}$ but all orders in $\Omega_{1}$ ) follows from

$$
\frac{r_{b c}(p)-r_{c b}(p)}{2 i}=\frac{\operatorname{Im}\left(\rho_{b c}^{0}\right) p}{p \Gamma+p^{2}+\left(\Omega_{1} / 2\right)^{2}}+\frac{\left(\rho_{a a}^{0}-\rho_{b b}^{0}\right) \Omega_{2} / 2}{p \Gamma+p^{2}+\left(\Omega_{1} / 2\right)^{2}}-\frac{\Omega_{2}}{2} \frac{\Gamma \rho_{a a}^{0}+\left(\rho_{b b}^{0}-1\right)(p-\Gamma)+2 \rho_{a a}^{0} p}{p(p+\Gamma)(p+2 \Gamma)+2(2 p+\Gamma)\left(\Omega_{1} / 2\right)^{2}} .
$$

It is worth looking at the partial contribution given by the first term on the right hand side of Eq. (16), namely $\operatorname{Im}\left(\rho_{b c}^{0}\right) p /\left[p \Gamma+p^{2}+\left(\Omega_{1} / 2\right)^{2}\right]$ for which the poles are

$$
p=-\frac{\Gamma}{2} \pm(i / 2) \sqrt{\Omega_{1}^{2}-\Gamma^{2}} .
$$

The inverse transform is easily found to give

$$
\begin{aligned}
\operatorname{Im}\left[\rho_{b c}(t)\right]= & \operatorname{Im}\left(\rho_{b c}^{0}\right) e^{-\Gamma t / 2} \cos \left(\frac{f t}{2}\right) \\
& -\operatorname{Im}\left(\rho_{b c}^{0}\right) e^{-\Gamma t / 2} \frac{\Gamma}{f} \sin \left(\frac{f t}{2}\right),
\end{aligned}
$$

where $f=\sqrt{\Omega_{1}^{2}-\Gamma^{2}}$. A similar result is given by $\mathrm{Li}$ and Xiao [14] and corresponds to standard damped Rabi nutation.

More generally, the contributions of $\rho_{a a}^{0}$ and $\rho_{b b}^{0}$ may not be ignorable, especially if $\operatorname{Im}\left(\rho_{b c}^{0}\right)$ is small or zero. Therefore we look at the full expression, Eq. (16), for which the first two terms are easily inverted. The denominator of the third term, however, is a cubic polynomial in $p$ and can be somewhat complicated. But as we are primarily interested in the case when the coupling field is strong, namely when $\Omega_{1} \gg \Gamma$, then the roots, $p_{1}$ and $p_{2}$, in the first two terms on the right hand side of Eq. (16) can be estimated as

$$
p_{1}=p_{2}^{*} \approx-\frac{\Gamma}{2}+i \frac{\Omega_{1}}{2},
$$

and those of the third term as

$$
\begin{aligned}
& p_{3} \approx-\frac{\Gamma}{2}, \\
& p_{4}=p_{5}^{*} \approx-\frac{5 \Gamma}{4}+i \Omega_{1},
\end{aligned}
$$

neglecting terms of order $\Gamma / \Omega_{1}$. Note that $p_{1}$ and $p_{2}$ give rise to damped oscillations of angular frequency $\Omega_{1} / 2$ as seen from Eq. (17), that $p_{3}$ yields a simple damped term, and that $p_{4}$ and $p_{5}$ give rise to damped oscillations of angular frequency $\Omega_{1}$. Using these roots, it is a matter of algebra to derive the following approximation to $\operatorname{Im}\left[\rho_{b c}(t)\right]$ :

$$
\operatorname{Im}\left[\rho_{b c}(t)\right]=\Phi_{12}(t)+\Phi_{3}(t)+\Phi_{45}(t),
$$

where 


$$
\begin{aligned}
\Phi_{12}(t) & \approx e^{-\Gamma t / 2}\left\{\operatorname{Im}\left(\rho_{b c}^{0}\right) \cos \left(\frac{\Omega_{1} t}{2}\right)+\frac{\Gamma}{\Omega_{1}}\left[\left(\rho_{a a}^{0}-\rho_{b b}^{0}\right) \frac{\Omega_{2}}{\Gamma}-\operatorname{Im}\left(\rho_{b c}^{0}\right)\right] \sin \left(\frac{\Omega_{1} t}{2}\right)\right\} \\
\Phi_{3}(t) & \approx-\frac{\Omega_{2}}{\Gamma} \frac{12\left(1-\rho_{b b}^{0}\right)}{9+\left(4 \Omega_{1} / \Gamma\right)^{2}} e^{-\Gamma t / 2} \\
\Phi_{45}(t) & \approx \frac{\Omega_{2}}{\Gamma} e^{-5 \Gamma t / 4}\left[\frac{12\left(1-\rho_{b b}^{0}\right)}{9+\left(4 \Omega_{1} / \Gamma\right)^{2}} \cos \left(\Omega_{1} t\right)+\frac{1-\rho_{b b}^{0}-2 \rho_{a a}^{0}}{2} \frac{\Gamma}{\Omega_{1}} \sin \left(\Omega_{1} t\right)\right]
\end{aligned}
$$

We have compared [33] the exact numerical inversion of Eq. (16) with the approximations in Eqs. (19) to (21). The agreement is qualitatively good even for values of $\Omega_{1} / \Gamma$ greater than 2 .

In Eq. (18), $\Phi_{12}(t)$ is the contribution of the poles at $p_{1}$ and $p_{2}$. That part of $\Phi_{12}(t)$ which is proportional to $\operatorname{Im}\left(\rho_{b c}^{0}\right)$ reduces in the limit of $\Omega_{1} \gg \Gamma$ to Eq. (17). $\Phi_{3}(t)$ results from the pole at $p_{3}$ and is strictly monotonically decreasing with time. $\Phi_{45}(t)$ results from poles $p_{4}$ and $p_{5}$ and oscillates (in the limit $\left.\Omega_{1} \gg \Gamma\right)$ twice as fast as $\Phi_{12}(t)$. In all the transient experimental and theoretical studies we know (including this present experimental study), the system behavior is dominated by $\Phi_{12}(t)$; however, should $\operatorname{Im}\left(\rho_{b c}^{0}\right)$ be small or zero with approximately equal ground state populations (i.e. $\rho_{a a}^{0} \approx \rho_{b b}^{0}$ ), then the faster oscillations of $\Phi_{45}$ might be observable. One way to do this would be to switch on both the probe and pump non-adiabatically at $t=0$. In this case we would have (in general) non-zero $\rho_{a a}^{0}, \rho_{b b}^{0}$ and $\rho_{c c}^{0}=1-\rho_{a a}^{0}-\rho_{b b}^{0}$, but vanishing off-diagonal components. A discussion of the faster oscillations using the 3D Vector Model [34, 35] appears in the Appendix.

Equations (17) to (21) were derived putting $\Gamma_{b a}=0$. In experimental realizations, a non-zero value of $\Gamma_{b a}$ can result from collisions, trap inhomogenieties and other effects. We can generalize Eq. (17) including this dephasing, to yield

$$
\begin{aligned}
\operatorname{Im}\left[\rho_{b c}(t)\right] \approx & -\operatorname{Im}\left(\rho_{b c}^{0}\right) e^{-t\left(\Gamma+\Gamma_{b a}\right) / 2} \frac{\Gamma-\Gamma_{b a}}{f^{\prime}} \sin \left(\frac{f^{\prime} t}{2}\right) \\
& +\operatorname{Im}\left(\rho_{b c}^{0}\right) e^{-t\left(\Gamma+\Gamma_{b a}\right) / 2} \cos \left(\frac{f^{\prime} t}{2}\right), \quad(22)
\end{aligned}
$$

where $f^{\prime}=\sqrt{\Omega_{1}^{2}-\left(\Gamma-\Gamma_{b a}\right)^{2}}$. We can also obtain the general forms of Eqs. (19) to (21) (for $\Omega_{1} \gg \Gamma$ ):

$$
\begin{aligned}
\Phi_{12}(t)= & e^{-t\left(\Gamma+\Gamma_{b a}\right) / 2}\left[\operatorname{Im}\left(\rho_{b c}^{0}\right)+\frac{\Omega_{2} \Gamma_{b a}}{3} \frac{5 \rho_{b b}^{0}+\rho_{a a}^{0}-2}{\Omega_{1}^{2}}\right] \cos \left(\frac{\Omega_{1} t}{2}\right) \\
& -\frac{\Omega_{2}}{\Omega_{1}} e^{-t\left(\Gamma+\Gamma_{b a}\right) / 2}\left[\operatorname{Im}\left(\rho_{b c}^{0}\right) \frac{\Gamma-\Gamma_{b a}}{\Omega_{2}}+\rho_{a a}^{0}-\rho_{b b}^{0}\right] \sin \left(\frac{\Omega_{1} t}{2}\right), \\
\Phi_{3}(t)= & -2 \frac{\Omega_{2}}{\Omega_{1}^{2}}\left[\Gamma_{b a}-\frac{3}{8}\left(1-\rho_{b b}^{0}\right)\left(\Gamma-4 \Gamma_{b a}\right) e^{-\Gamma t / 2}\right], \\
\Phi_{45}(t)= & \frac{\Omega_{2}}{\Omega_{1}} e^{-5 \Gamma t / 4}\left[\left(\frac{3 \Gamma}{4} \frac{1-\rho_{b b}^{0}}{\Omega_{1}}+\frac{\Gamma_{b a}}{3} \frac{1-2 \rho_{a a}^{0}-\rho_{b b}^{0}}{\Omega_{1}}\right) \cos \left(\Omega_{1} t\right)+\frac{1-2 \rho_{a a}^{0}-\rho_{b b}^{0}}{2} \sin \left(\Omega_{1} t\right)\right] .
\end{aligned}
$$

\section{Turn-off transient for arbitrary detunings of pump and probe}

The turn-off case is relatively simple as only the probe field acts during the transient period. Thus we are concerned with optical pumping by the probe, as considered earlier, but in this case the initial values are different and we shall work to first order in the probe field $\Omega_{2}$. We suppose that both the coupling and probe fields have been turned on for a time long compared to $\Gamma^{-1}$ and the coupling field is then switched off at a time now taken to be $t=0$. We find [33] that the inverse Laplace transform of $\rho_{b c}(t)$ is

$$
r_{b c}(p)=\frac{1}{p+\Gamma+i \Delta_{2}}\left[\rho_{b c}^{0}+i \frac{\Omega_{2}}{2} \frac{(p-\Gamma)\left(1-\rho_{a a}^{0}\right)-\rho_{b b}^{0}(2 p+\Gamma)}{p(p+2 \Gamma)}\right]+O\left(\frac{\Omega_{2}}{\Gamma}\right)^{3} .
$$


The initial values $\rho_{a a}^{0}, \rho_{b b}^{0}$ and $\rho_{b c}^{0}$ are found by solving for the $r_{\alpha \beta}(p)$ during the epoch when both fields act, and then taking the long-time limits, Eq. (12). Thus we have

$$
\begin{aligned}
& \rho_{a a}^{0}=O\left(\frac{\Omega_{2}}{\Gamma}\right)^{2}, \\
& \rho_{b b}^{0}=1+O\left(\frac{\Omega_{2}}{\Gamma}\right)^{2}, \\
& \rho_{b c}^{0}=\frac{\Omega_{2}}{2} \frac{\Delta_{21}-i \Gamma_{b a}}{\left(\frac{\Omega_{1}^{2}}{4}-\Delta_{2} \Delta_{21}+\Gamma_{b a} \Gamma\right)+i\left(\Delta_{21} \Gamma+\Delta_{2} \Gamma_{b a}\right)}+O\left(\frac{\Omega_{2}}{\Gamma}\right)^{3},
\end{aligned}
$$

so that,

$$
r_{b c}(p)=\frac{1}{p+\Gamma+i \Delta_{2}}\left(\rho_{b c}^{0}-i \frac{\Omega_{2}}{p}\right)+O\left(\frac{\Omega_{2}}{\Gamma}\right)^{3}
$$

This expression contains simple poles at $p=0$ and $p=-\Gamma-i \Delta_{2}$ so that for $t \geq 0$ one has

$$
\rho_{b c}(t) \approx \rho_{b c}^{0} e^{-\Gamma t} e^{-i \Delta_{2} t}-i \frac{\Omega_{2}}{2}\left(\frac{1-e^{-\Gamma t} e^{-i \Delta_{2} t}}{\Gamma+i \Delta_{2}}\right) .
$$

Finally, using Eq. (23) for $\rho_{b c}^{0}$, and taking the imaginary part of $\rho_{b c}(t)$, gives to order $\Omega_{2} / \Gamma$

$$
\begin{aligned}
\operatorname{Im}\left[\rho_{b c}(t)\right]= & -\frac{\Omega_{2}}{2} \frac{\Gamma+e^{-\Gamma t}\left[\Delta_{2} \sin \left(\Delta_{2} t\right)-\Gamma \cos \left(\Delta_{2} t\right)\right]}{\Gamma^{2}+\Delta_{2}^{2}} \\
& -\frac{\Omega_{2}}{2} e^{-\Gamma t} \frac{\left[\Gamma \Delta_{21}^{2}+\Gamma_{b a}\left(\Gamma \Gamma_{b a}+\Omega_{1}^{2} / 4\right)\right] \cos \left(\Delta_{2} t\right)}{\left(\Gamma \Gamma_{b a}-\Delta_{2} \Delta_{21}+\Omega_{1}^{2} / 4\right)^{2}+\left(\Delta_{21} \Gamma+\Delta_{2} \Gamma_{b a}\right)^{2}} \\
& -\frac{\Omega_{2}}{2} e^{-\Gamma t} \frac{\Delta_{21}\left[\frac{\Omega_{1}^{2}}{4}-\Delta_{2}\left(\Delta_{21}+\Gamma_{b a}^{2} / \Gamma\right)\right] \sin \left(\Delta_{2} t\right)}{\left(\Gamma \Gamma_{b a}-\Delta_{2} \Delta_{21}+\Omega_{1}^{2} / 4\right)^{2}+\left(\Delta_{21} \Gamma+\Delta_{2} \Gamma_{b a}\right)^{2}}
\end{aligned}
$$

which is the required turn-off transient. It is interesting to note that $\Gamma_{b a}$ enters Eqs. (24) and (25) only through the initial conditions. This may be understood by recalling that $\Gamma_{b a}$ refers to a two photon dephasing, and so will not be dynamically important when only the probe field is acting. We note that, at $t=0$ (the instant of turn-off) one has, from Eqs. (23) or (25),

$$
\operatorname{Im}\left(\rho_{b c}^{0}\right)=-\frac{\Omega_{2}}{2} \frac{\Gamma \Delta_{21}^{2}+\Gamma_{b a}\left(\Gamma \Gamma_{b a}+\Omega_{1}^{2} / 4\right)}{\left(\Gamma \Gamma_{b a}-\Delta_{2} \Delta_{21}+\Omega_{1}^{2} / 4\right)^{2}+\left(\Delta_{21} \Gamma+\Delta_{2} \Gamma_{b a}\right)^{2}}
$$

which is the general expression for the steady-state EIT lineshape for arbitrary detunings.

\section{Overview of transient response}

We now present an overview of the turn-on and turnoff transients displayed as pseudocolour plots showing $\operatorname{Im}\left[\rho_{b c}(t)\right]$ as a function of time $t$ and probe detuning $\Delta_{2}$. The parameters are chosen to correspond closely to the experimental conditions described in detail in the following sections. We use the spontaneous radiative decay rate $(2 \Gamma=5.68 \mathrm{MHz})$ and the effects of uncoupled absorptions are not included in the model. (Uncoupled absorptions are extra absorptions which derive from degenerate energy levels: see for example 36 for a full description). In order to generate the pseudocolour plots, we numerically turned on the probe field at a finite negative time $(t=-10 \Gamma)$ which was sufficient to build up a steady state $\rho_{b c}^{0}$. Initial populations (at $t=-10 \Gamma$ ) were chosen so 


\section{$\Delta_{1}=0 \mathrm{MHz}$}
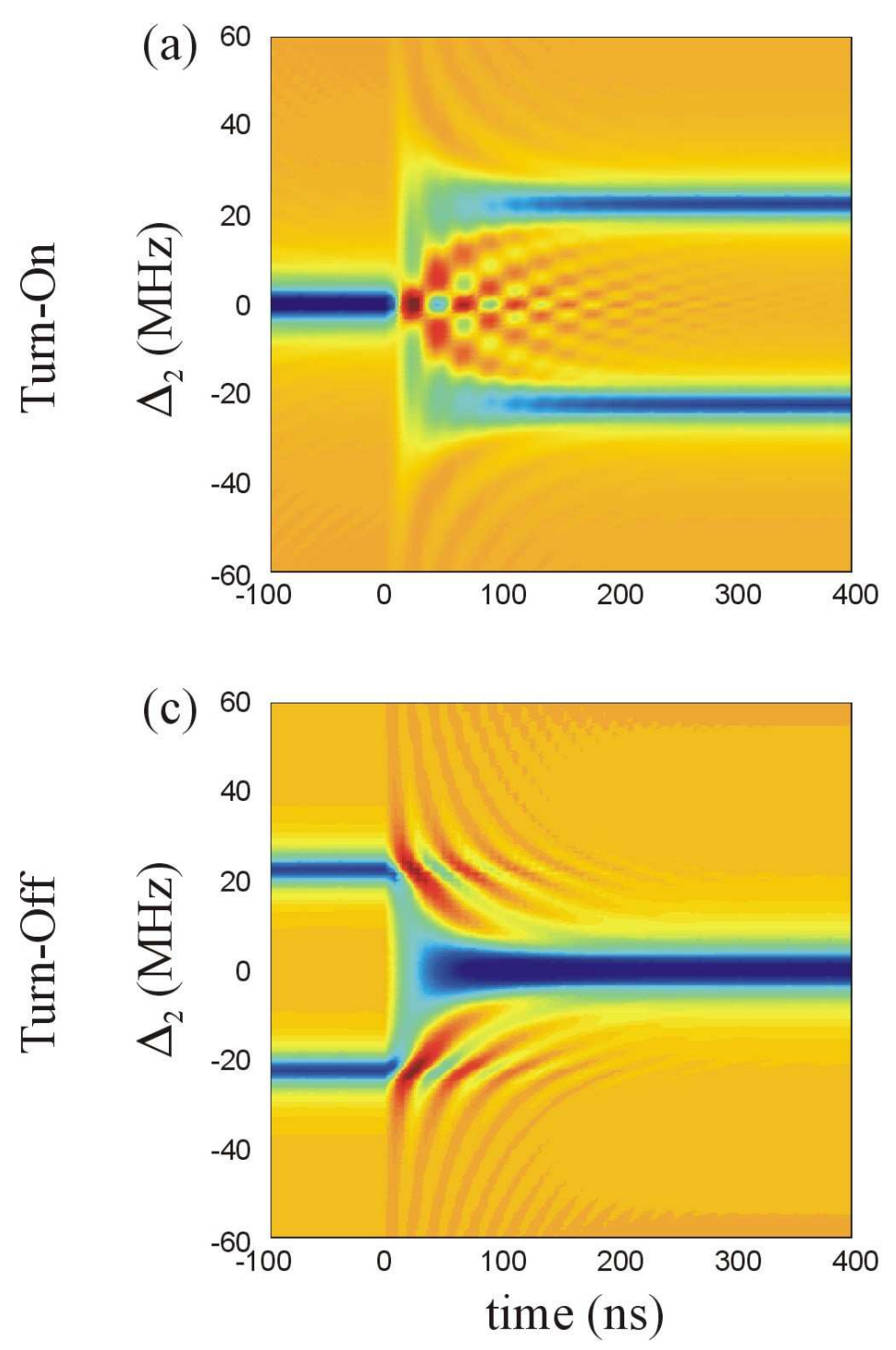

$\Delta_{1}=-23 \mathrm{MHz}$

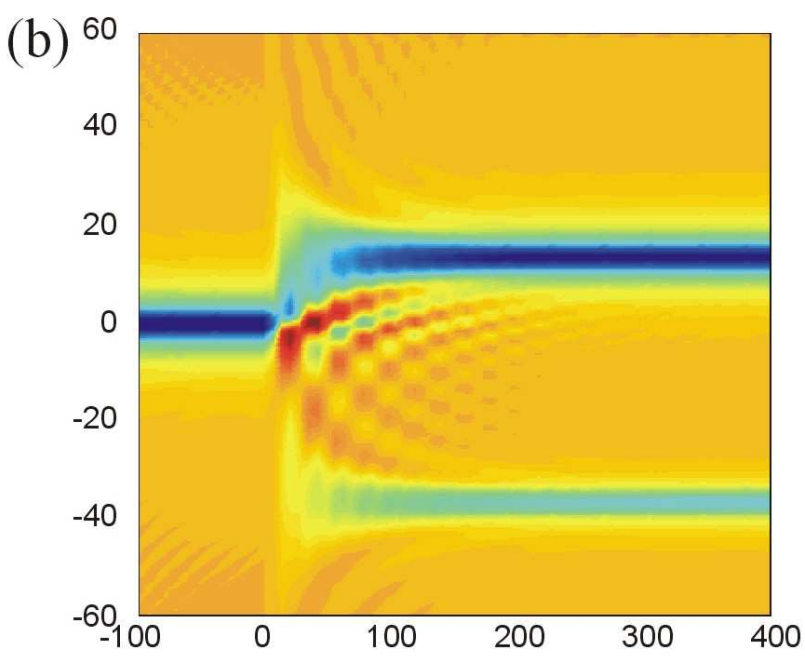

(d)

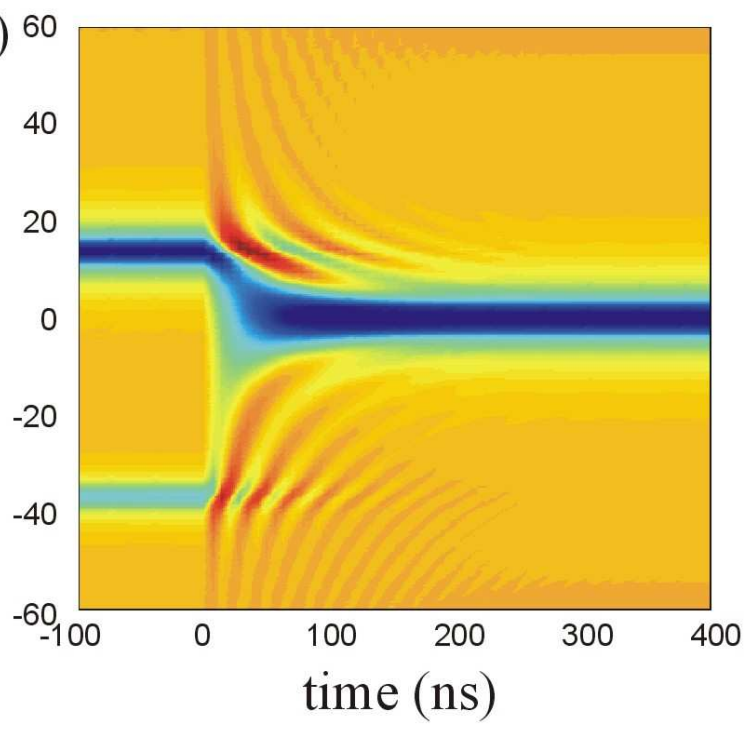

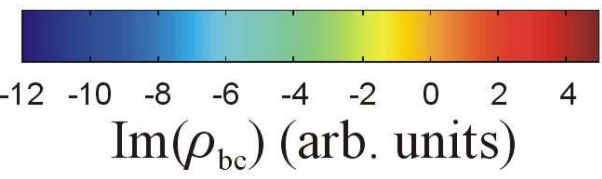

FIG. 2: Pseudocolour plots of $\operatorname{Im}\left[\rho_{b c}(t)\right]$ as a function of time, $t$, and probe detuning, $\Delta_{2}$ for (a) turn-on; resonant coupling field: $\Delta_{1}=0 \mathrm{MHz}$, (b) turn-on; detuned coupling field: $\Delta_{1}=-23 \mathrm{MHz}$ (c) turn-off; resonant coupling field: $\Delta_{1}=0 \mathrm{MHz}$ and (d) turn-off; detuned coupling field: $\Delta_{1}=-23 \mathrm{MHz}$. In all cases we have used: $\Omega_{1}=45 \mathrm{MHz}, \Omega_{2}=1 \mathrm{MHz}, \Gamma=5.68 \mathrm{MHz}$ and $\Gamma_{b a}=3.4 \mathrm{MHz}$. (The effect of uncoupled absorptions are not included in these plots.)

that the populations at time $t=0$ corresponded closely to the experimental conditions $\left(\rho_{a a}(t=-10 \Gamma)=0.05\right.$, $\left.\rho_{b b}(t=-10 \Gamma)=0.95\right)$. We term this the 'linear regime' as small variations will only introduce extra global scaling factors to the amplitude of the probe response.

The turn-on transients are shown in FIGS. 2(a) and (b), where the coupling field is switched on at $t=0$.
We consider first the case of a resonant coupling field, FIG. 2(a). Scanning the entire figure from left to right, we first see (for $t<0$ ) the Lorentzian probe absorption feature of spectral width $2 \Gamma$ as the probe drives the bare $|b\rangle-|c\rangle$ transition. Then in the transient regime from $t=0$ to several $\Gamma^{-1}$, we find modified Rabi nutations due primarily to the coupling of the ground state $|b\rangle$ to 
the dressed states $| \pm\rangle=(|a\rangle \pm|c\rangle) / \sqrt{2}$. If the probe were to couple $|b\rangle$ to a single dressed state, say $|+\rangle$, it would undergo decaying Rabi oscillations at the detuned frequency $\sqrt{\left(\Delta_{2}+\Omega_{1} / 2\right)^{2}+\Omega_{2}^{2}}$. The peaks of these oscillations would lie on the curves $\Delta_{2}= \pm 2 \pi n / t+\Omega_{1} / 2$ for $t \ll 2 \pi / \Omega_{2}$ and $n=1,2, \ldots$ Oscillations of this kind are clearly evident in FIG. 2(a) for each of the dressed states. However, the fact that the probe couples $|b\rangle$ to both dressed states simultaneously, evidently, results in interference between the Rabi oscillations due to the probe interacting with each dressed state separately. This suggests that the central pattern in FIG. 2(a) represents transient dressed-state interferences. In addition, for $\Delta_{2} \approx 0$, the preparation of the system before the coupling field is switched on induces relatively strong absorption and a larger value of $\operatorname{Im}\left(\rho_{b c}\right)$ at $t=0$. This leads to enhanced Rabi nutations along the $\Delta_{2}=0$ line for $t>0$. Finally, for long times, we see the two well-resolved absorption features, each of width $\Gamma$, corresponding to the well known Autler-Townes doublet [37. We note that our pseudocolour plots show a superficial similarity to the three dimensional plots presented by $\mathrm{Lu}$ et al 12 but the different initial conditions between the two cases studied result in quite different dynamics.

We now focus on the case where the probe too is fixed on resonance, i.e. the time-line $\Delta_{2}=0$ in FIG. 2(a). Here, for $t>0$, we see gain and absorption cycles of the kind discussed in refs [14, 16] and observed by us previously 22, 23. For a strong coupling field $\left(\Omega_{1} / \Gamma \gg 1\right)$ this transient $\left(\Delta_{2}=0\right)$ is well described by Eq. (22). Two other lines of note are at $\Delta_{2}= \pm \Omega_{1} / 2$ where transparency for $t<0$ changes transiently to absorption as the probe begins to resonantly monitor a transition to a dressed state.

Figure 2(b) shows the case of a detuned coupling field. Again, we observe a transient regime featuring absorption and gain regions evolving to the resolved dressed state absorption features centered at $\Delta_{2}=\Delta_{1} / 2 \pm$ $\sqrt{\left(\Omega_{1}^{2}+\Delta_{1}^{2}\right) / 4}$, but in this case we can clearly distinguish between the dominant or major dressed state and the narrower minor dressed state. In the bare state picture, in the limit of large $\Delta_{1}$ these two features are associated, respectively, with one-photon absorption $(|b\rangle-|c\rangle)$ and two-photon absorption $(|b\rangle-|c\rangle-|a\rangle)$.

We now consider the turn-off transients shown in FIGS. 2(c) and (d), where both fields are assumed to have been on for all negative times to establish the initial conditions, and the coupling field is switched off at $t=0$. The dominant feature in the transient regime is the $1 / t$ broadened absorption region where the probe begins to drive the bare transition. Of more interest are the strong transient gain peaks that can be seen along the two lines $\left.\Delta_{2}=\Delta_{1} / 2 \pm \sqrt{\left(\Omega_{1}^{2}+\Delta_{1}^{2}\right) / 4}\right)$ at the levels associated with the two dressed state transitions before turn-off. This is simply the off-resonant probe monitoring the relaxation of a two-level atom with specially prepared initial conditions. We note that these tran-

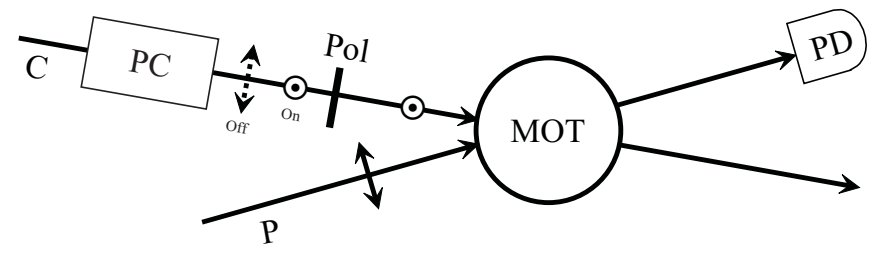

FIG. 3: Schematic of experimental beam configuration. The coupling, $C$, and probe, $P$, beams make an angle of about $20^{\circ}$. $\mathrm{PD}$ is an avalanche photodiode, $\mathrm{PC}$ is a Pockels Cell and Pol a polariser. $P$ is linearly polarised in the horizontal plane while $C$ is linearly polarised in the vertical plane when the $\mathrm{PC}$ is on and linearly polarised in the horizontal plane when the $\mathrm{PC}$ is off.

sient gain regions are tunable by varying the detuning and strength of the coupling beam, and are remote from any uncoupled absorptions in a real $\Lambda$ system that might exist in the $\Delta_{2}=0$ region [36, 38]. We note also that the entire $\left(t, \Delta_{2}\right)$ region of this figure is described by Eq. (25).

\section{EXPERIMENTAL SET-UP AND PROCEDURE}

The $\Lambda$ system was realized in a $\mathrm{Rb}^{87}$ MOT substantially similar to the one described in [23]. The sample contained about $5 \times 10^{7}$ atoms in a region of diameter about $3 \mathrm{~mm}$. The relevant energy levels and excitation scheme are shown in FIG. 1 (b). A schematic showing the layout of coupling and probe beams is shown in FIG. 3. The coupling beam had an average intensity $\approx 100 \mathrm{~mW} / \mathrm{cm}^{2}$ with a roughly elliptical profile $2 \mathrm{~mm} \times 4 \mathrm{~mm}$. The probe beam had an average intensity $\approx 0.03 \mathrm{~mW} / \mathrm{cm}^{2}$ with circular profile of diameter about $1 \mathrm{~mm}$. The beams propagated in the sample with orthogonal linear polarizations and with an angle of about $20^{\circ}$ between their directions of propagation. All laser beams were derived from external-cavity laser diodes, using master-slave injection where appropriate. Non-adiabatic switching of the coupling field was realized using a Linos Photonics LM10 Pockels cell with a custom designed high voltage switch. The rise time of the switch and associated detection circuitry was less than $6 \mathrm{~ns}$. The excited $5 P_{3 / 2}$ state lifetime is $28 \mathrm{~ns}$ and the relevant Rabi periods are of similar magnitude and so the non-adiabatic condition is fairly well satisfied. Because the fall time of the switch is very long, the Pockels cell could only be operated in either fast turn-on or fast turnoff mode, necessitating separate experiments to observe turn-on and turn-off transients. Changing between turnon and turn-off experiments was realized by rotating the plane of polarization of the coupling field by $90^{\circ}$ before entering the Pockels cell.

The probe detuning was set by varying the cavity size of an external-cavity grating-controlled diode laser (ECDL) using the voltage offset of the piezo-electric transducer, which was monitored on a voltmeter. This 
voltage was calibrated using the saturated absorption spectrum in a standard $\mathrm{Rb}$ cell. Drifts in the cavity size due to temperature fluctuations were checked and corrected for by determining the voltage which put the probe field on resonance at the beginning of each transient experiment. Nevertheless, the absolute detuning of the probe field could not be determined to an accuracy better than about half a linewidth $(\approx 3 \mathrm{MHz})$, except when the field was locked to resonance. Standard detunings used were generally much larger than $3 \mathrm{MHz}$, with dressed state detunings being as large as $36 \mathrm{MHz}$. The measured transients were stored in a Tektronix TDS520B digital oscilloscope. Instabilities in our control method limited the maximum number of averages to about 50 . Because of small drifts in the intensities of our laser diodes (which were run in constant current mode for maximum frequency and mode stability), probe transmission levels were measured with the MOT turned off between transient runs and the transmission level at the time of the experiment inferred using a linear regression.

Our experiments were carried out in the optically thin regime with maximum probe absorption of about $15 \%$ at resonance. The probe Rabi frequency of about $4 \mathrm{MHz}$ gave a good signal to noise ratio without producing any measurable power broadening. Under these conditions the probe absorption increases linearly with probe intensity and it was found convenient to normalize our probe absorption/transmission levels to the steady state absorption in the absence of the coupling field. We use the same method as that described in 23. where we define the maximum probe absorption in the absence of a coupling field as the zero transmission level. Thus, if we define the voltage measured on our photodetector as $\mathcal{V}$, the signal for maximum probe absorption (minimum probe transmission) $\mathcal{V}_{\text {min }}$ and the signal without any probe absorption $\mathcal{V}_{0}$, then our scaled transmission levels for a detected signal are defined by

$$
T=\frac{\mathcal{V}-\mathcal{V}_{\min }}{\mathcal{V}_{0}-\mathcal{V}_{\min }}
$$

This definition for the transmission has the advantage of being simple and robust against day-to-day fluctuations in the number of atoms trapped in our sample (provided we are in a linear absorption regime). It is also easy to make direct comparisons with theory by noting that any detected changes in signal level will be proportional to $\operatorname{Im}\left(\rho_{b c}\right)$, with values of $T>1$ associated with gain and $T<1$ indicating absorption.

With on-resonant or near-resonant probe fields our experimental transients are accompanied by the timeindependent effects of uncoupled absorptions. These have been fully described in our previous paper [36]. For the purposes of this work it suffices to treat the system as an ensemble of identical three level atoms in an ideal $\Lambda$ configuration, as in Section I, together with a similar ensemble that does not interact with the coupling field. In our turn-on experiments, uncoupled absorptions were found to contribute about $20 \%$ of the maximum reso- nant absorption and are assumed to have a Lorentzian line shape centered at zero probe detuning.

\section{EXPERIMENTAL RESULTS AND DISCUSSIONS}

We first present experimental steady-state probe frequency spectra (EIT traces) for a resonant and an offresonant coupling field with the same parameters that apply in the turn-on transient experiments. The central region of FIG. 4 (a) shows the probe spectrum for the resonant coupling field. The dressed state absorption peaks are clearly resolved on either side of the uncoupled absorption at $\Delta_{2}=0$. The Rabi frequency of the coupling field can be inferred from this figure to be $\Omega_{1}=45 \mathrm{MHz}$. This value is consistent with that obtained from beam parameters and absorption line strengths. Also seen are the absorption peaks on the $5 S_{1 / 2} F=2$ to $5 P_{3 / 2} F=1$ transition at $\Delta_{2}=-157 \mathrm{MHz}$ and the $5 S_{1 / 2} F=2$ to $5 P_{3 / 2} F=3$ transition at $\Delta_{2}=267 \mathrm{MHz}$. These peaks were used for frequency calibration purposes. Figure $4(\mathrm{~b})$ shows the probe spectrum when the coupling field is detuned by $\Delta_{1}=-23 \mathrm{MHz}$. The major dressed state absorption peak is seen at $\Delta_{2}=14 \mathrm{MHz}$ and the minor one at $\Delta_{2}=-37 \mathrm{MHz}$. Uncoupled absorptions are barely resolved in the wing of the major dressed state. We note that the linewidths in these spectra are approximately $11 \mathrm{MHz}$, or nearly twice the spontaneous decay rate of the $5 P_{3 / 2}$ levels. We attribute this additional broadening to inhomogeneities in the sample principally due to light shifts caused by the coupling beam and the trapping beams with smaller contributions from laser linewidths. Accordingly we have taken a phenomenological value for the half linewidth of $\Gamma=5.5 \mathrm{MHz}$ in our theoretical transients rather than the standard value of $2.8 \mathrm{MHz}$, as discussed in 36. The two-photon dephasing rate is taken to be $\left.\Gamma_{b a}=0.6 \Gamma 22\right]$.

We now present experimental turn-on and turn-off transients for the resonant and the non-resonant coupling field, together with the corresponding theoretical curves. Turn-on theory transients were obtained by numerically solving Eqs. (11). Turn-off theory transients were obtained by fitting the experimental data to Eq. (25). In each case representative selections of probe detunings are chosen so that horizontal lines across FIGS. 2(a)-(d) are explored.

When we compare the experimental and theoretical curves in FIGS. 58, we find excellent agreement in the transient behavior. We therefore use our theoretical results to determine $\Delta_{2}$ which we find to be consistent with detunings derived from the piezo-offset. It has been less easy to make direct comparisons of the overall transmission levels and there are minor inconsistencies when comparing experimental with theoretical levels in our transients. We believe that these inconsistencies are due to difficulties in correcting for all of the intensity fluctuations and drifts in our lasers on the timescales of our 
(a)
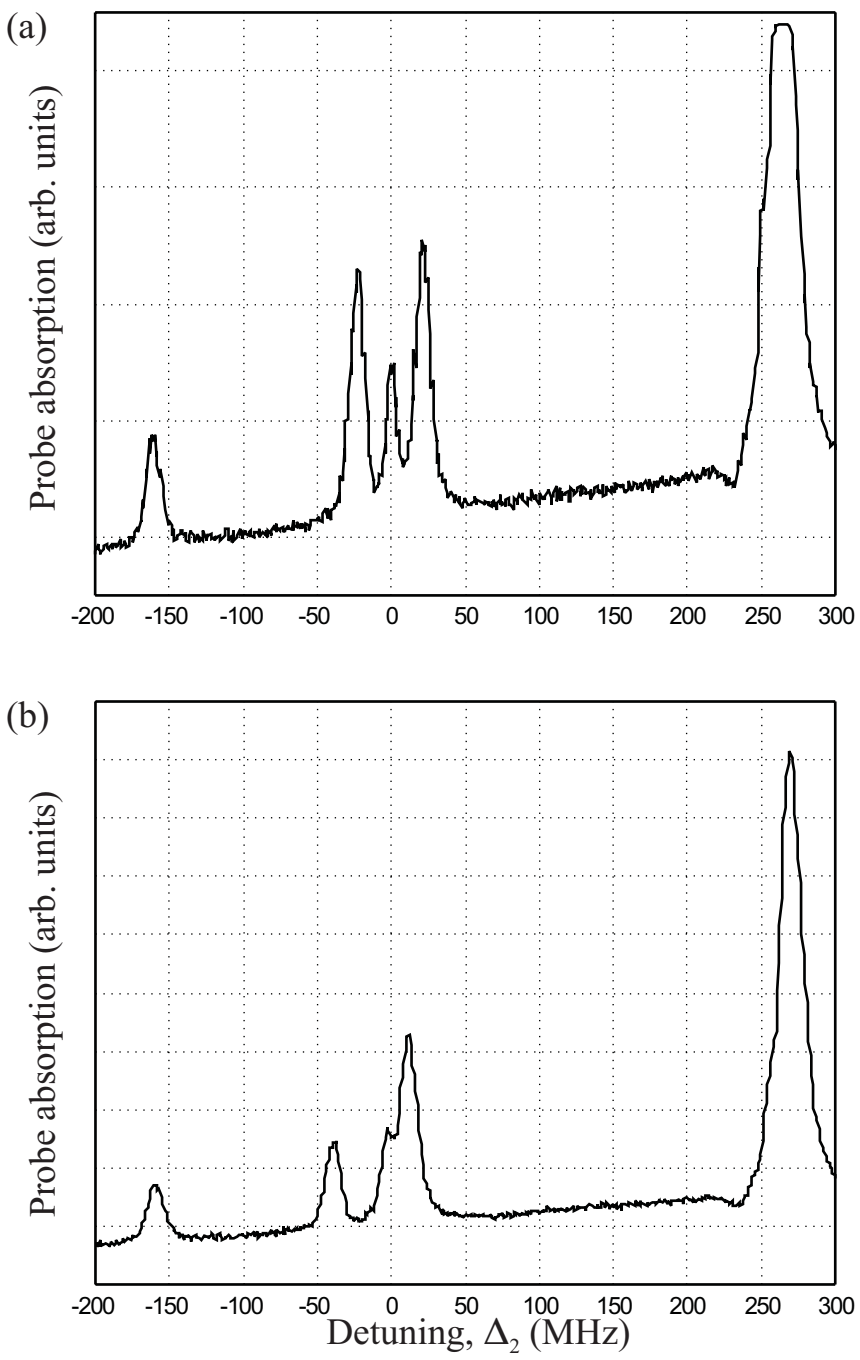

FIG. 4: Steady state probe transmission spectra. (a) Onresonance coupling field with Rabi frequency $\Omega_{1}=45 \mathrm{MHz}$ showing dressed state absorptions at detunings $\Delta_{2}= \pm \Omega_{1} / 2$ and uncoupled absorption at $\Delta_{2}=0$. (The peak on the $5 S_{1 / 2}, F=2$ to $5 P_{3 / 2}, F=3$ transition has been truncated to fit on the page). (b) Off-resonant coupling excitation with Rabi frequency $\Omega_{1}=45 \mathrm{MHz}$ and detuning $\Delta_{1}=-23 \mathrm{MHz}$. The major dressed state is at $\Delta_{2}=14 \mathrm{MHz}$ and the minor one at $\Delta_{2}=-37 \mathrm{MHz}$. Uncoupled absorptions are barely resolved in the wing of the major dressed state. In each case note the presence of extra absorption peaks corresponding to the $5 S_{1 / 2}, F=2$ to $5 P_{3 / 2}, F=1$ and $5 S_{1 / 2}, F=2$ to $5 P_{3 / 2}, F=3$ transitions at $\Delta_{2}=-157 \mathrm{MHz}$ and $\Delta_{2}=267 \mathrm{MHz}$ respectively for frequency calibration purposes. Each trace is an average over 50 scans. Day to day fluctuations in signal levels mean that the absorption scales in (a) and (b) are different.

experiments, especially as they relate to mode hops in the probe laser as it was detuned.

\section{A. Turning on the coupling field}

For these experiments the coupling field is turned on at time zero after the probe field and MOT fields have been on for a long time. The left-hand traces in FIGS. F(a)-(c) show the experimental results for turning on a resonant coupling field $\left(\Delta_{1}=0\right)$ of Rabi frequency $\Omega_{1}=45 \mathrm{MHz}$. The right hand traces show the corresponding theory curves, with the steady transmission levels adjusted to take account of the uncoupled absorption. Figure 5 (a) shows the well-known case of zero probe detuning $\left(\Delta_{2}=0\right)$ exhibiting significant gain $(T=1.35)$ despite the presence of uncoupled absorption. This curve is substantially similar to FIG. 3(b) of 23]. The longtime transmission settles down to the value of $T=0.8$ showing the effects of uncoupled absorption as well as effects due to the phenomenological two-photon dephasing rate. In FIG. 5(c) the probe detuning is $20 \mathrm{MHz}$ and so the probe transmission is almost unity before turn-on. After turn-on, the transmission falls transiently as the probe begins to resonantly couple the dressed state. The long-time transmission is close to the value 0.5 which is expected from the equal absorption strengths of the two dressed states. We term this phenomenon, dressed state filling, because the increase in probe absorption and the associated movement of coherences and populations are accompanying the transient dressing of the $|a\rangle-|c\rangle$ transition. This has previously been called three-level freeinduction decay 10]. Figure 5(b) shows an intermediate case with probe detuning of $\Delta_{2}=8 \mathrm{MHz}$. No significant differences were found between negative and positive detunings of the probe, as expected from the theory, and so negative detuning traces are not shown.

We now consider the case where the coupling field is detuned from resonance by $\Delta_{1}=-23 \mathrm{MHz}$. Other parameters and the dynamics before turn-on are the same as before. Experimental and theoretical transient results are presented in FIGS. 6(a) to (e). The first three figures show (a) the case of a resonant probe, (b) the filling of the major dressed state $\left(\Delta_{2}=15 \mathrm{MHz}\right)$, and (c) the filling of the minor dressed state $\left(\Delta_{2}=-37 \mathrm{MHz}\right)$. Trace (d) $\left(\Delta_{2}=-6 \mathrm{MHz}\right)$ shows the case where the probe is detuned just to the red of the bare state absorption line. It is interesting because of the clarity of the signal obtained coupled with the relatively high gain $(T \approx 1.26)$ making this a promising region for further transient gain experiments. Finally in FIG. 6(e) $\left(\Delta_{2}=9 \mathrm{MHz}\right)$ the probe is detuned by a similar amount to the blue and shows enhanced absorption dips.

\section{B. Turning off the coupling field}

The experimental conditions for turn-off were different in some respects from those for turn-on. The maximum coupling-field intensity passing the Pockels cell for turnoff was $\Omega_{1}=46 \mathrm{MHz}$, slightly greater than that for turnon; also the uncoupled absorption levels are slightly dif- 
(a)

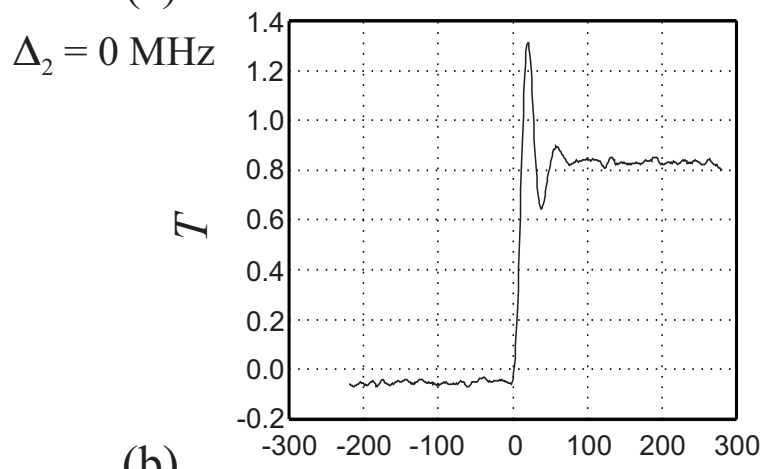

(b)

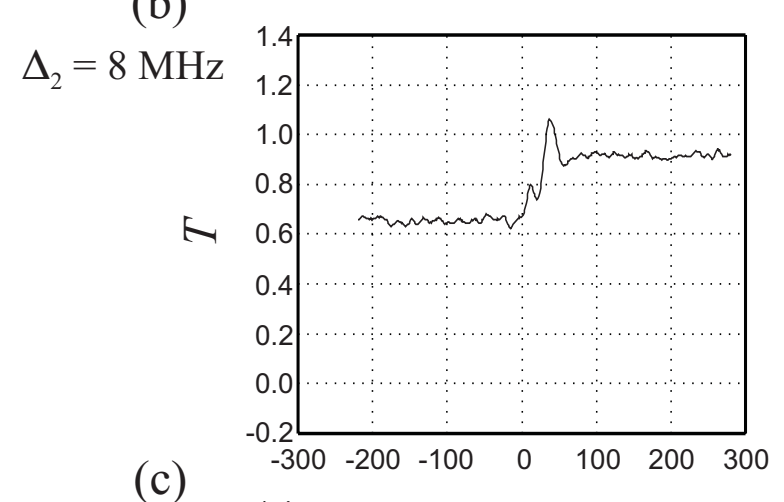

(c)

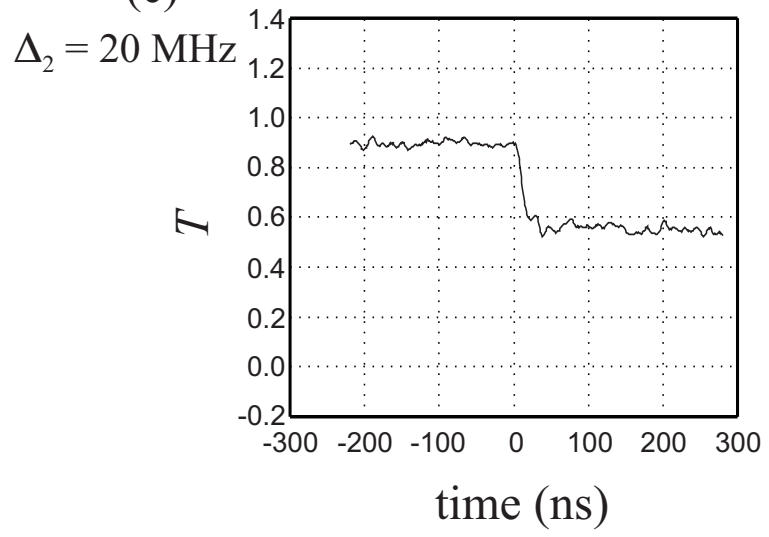

Experiment
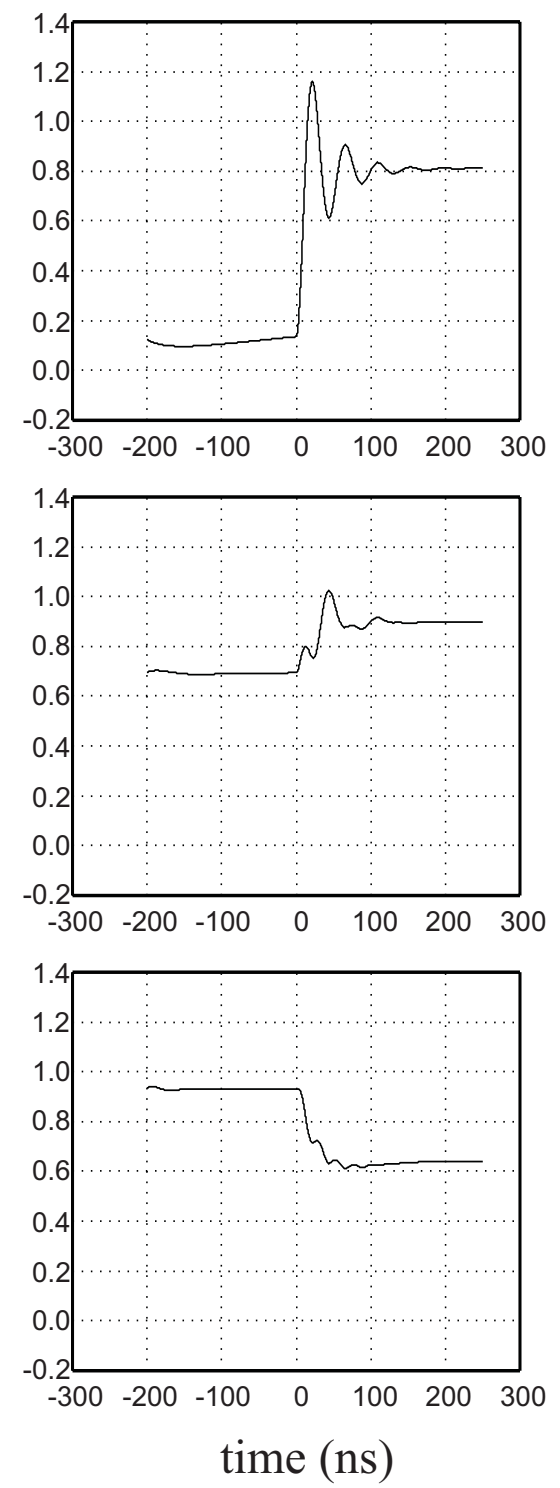

FIG. 5: Turn-on transients for varying probe detunings with a resonant coupling field: (a) $\Delta_{2}=0 \mathrm{MHz}$ (resonant probe), (b) $\Delta_{2}=8 \mathrm{MHz}$, and (c) $\Delta_{2}=20 \mathrm{MHz}$ (dressed state filling). In all cases $\Omega_{1}=45 \mathrm{MHz}$ and 50 averages were taken.

ferent, probably as a result of optical pumping amongst the Zeeman substates by the coupling field before turnoff. We have found that some of the experimental turn-off transients are remarkable for the high gain and clarity of signal obtained. We attribute the clearer signals to the fact that the system is simpler without the inhomogeneous light shift effects of the coupling field.

Using the analytical result, Eq. (25) with the addition of the effects of uncoupled absorption, we have been able to perform fits to the experimental data using Origin 6.0284 [39]. The results of these fits are presented as the theoretical traces. The interdependence of many of the parameters mean that some parameters cannot be adequately determined using this method. Fortunately the probe detuning, $\Delta_{2}$, can be well determined using this method, and the agreement between experiment and theory shows that our system is well described by our three level analysis. Except where mentioned, the values for $\Delta_{2}$ quoted below were determined using the curve fitting.

The experimental and corresponding theoretical transients for a resonant coupling field are presented in FIGS. B(a) to (d). Here $t=0$ defines the turn-off time. Case (a) for a resonant probe shows the transmission dropping from the uncoupled absorption level of $T \approx 0.6$ to $T=0$ as the probe fills the bare state transition. Of more interest is case (b) where the probe is detuned to a level corresponding to a pre turn-off dressed state $\left(\Delta_{2}=-22 \mathrm{MHz} \simeq-\Omega_{1} / 2\right)$. We term this dressed state emptying by comparison with the dressed state filling re- 
(a)

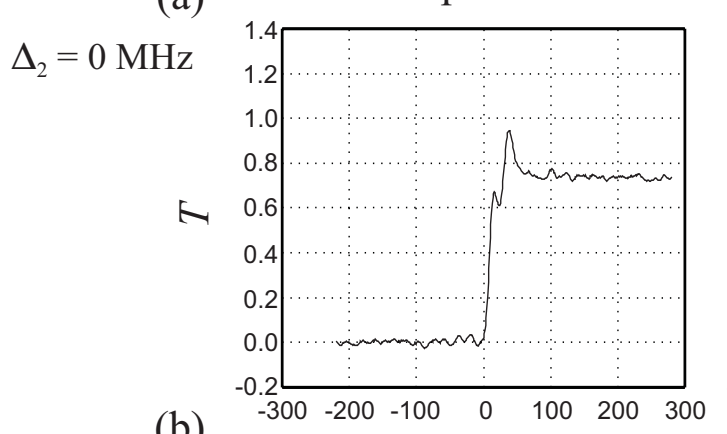

(b)

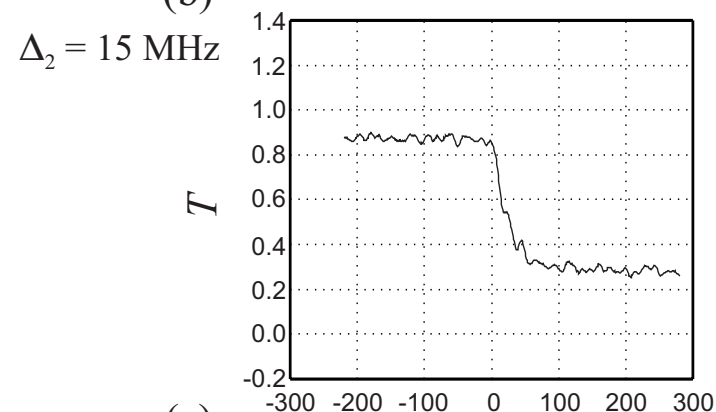

(c)

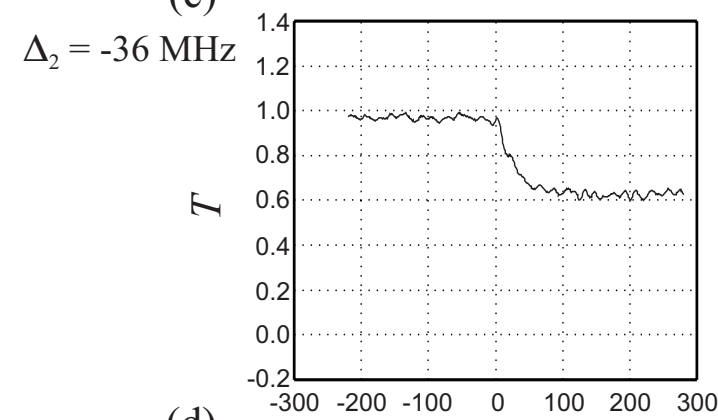

(d)

$$
\Delta_{2}=-6 \mathrm{MHz}
$$

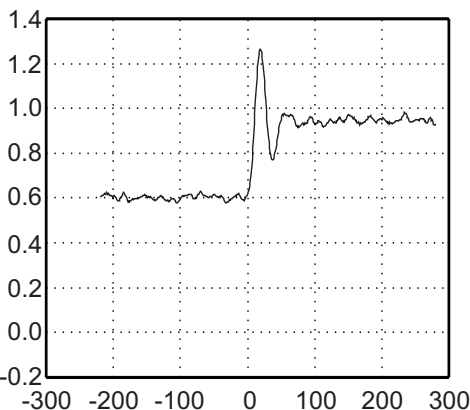

(e)

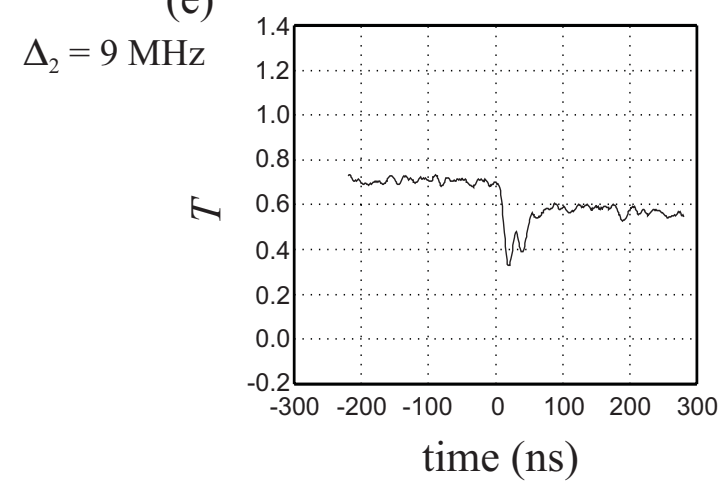

Theory
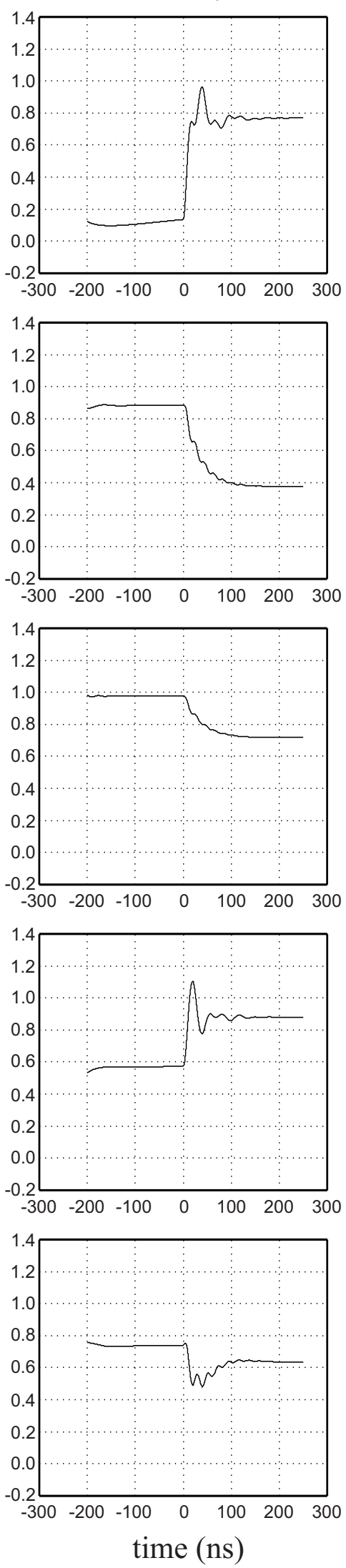

FIG. 6: Turn-on transients for varying probe detunings with an off-resonant coupling field: (a) $\Delta_{2}=0 \mathrm{MHz}$ (resonant probe), (b) $\Delta_{2}=15 \mathrm{MHz}$ (major dressed state filling), (c) $\Delta_{2}=-36 \mathrm{MHz}$ (minor dressed state filling) (d) $\Delta_{2}=-6 \mathrm{MHz}$ and (e) $\Delta_{2}=9 \mathrm{MHz}$. In all cases $\Omega_{1}=45 \mathrm{MHz}, \Delta_{1}=-23 \mathrm{MHz}$ and 50 averages were taken. 
(a)

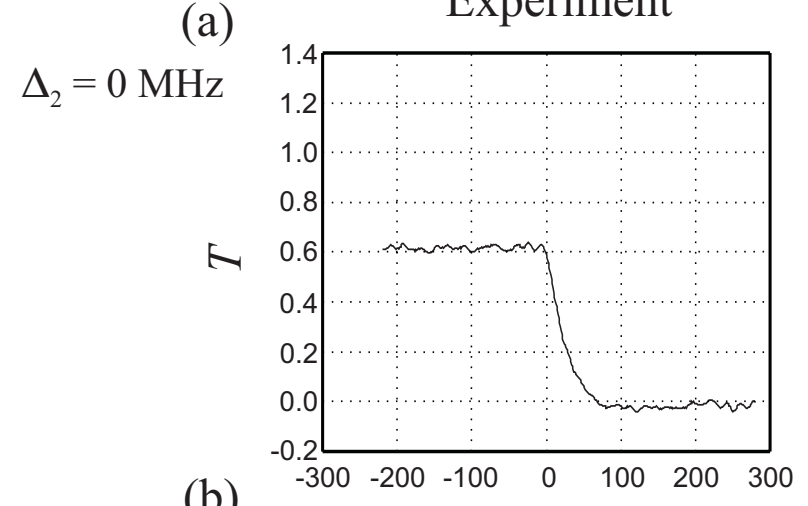

(b)

$\Delta_{2}=22 \mathrm{MHz}$

(c)

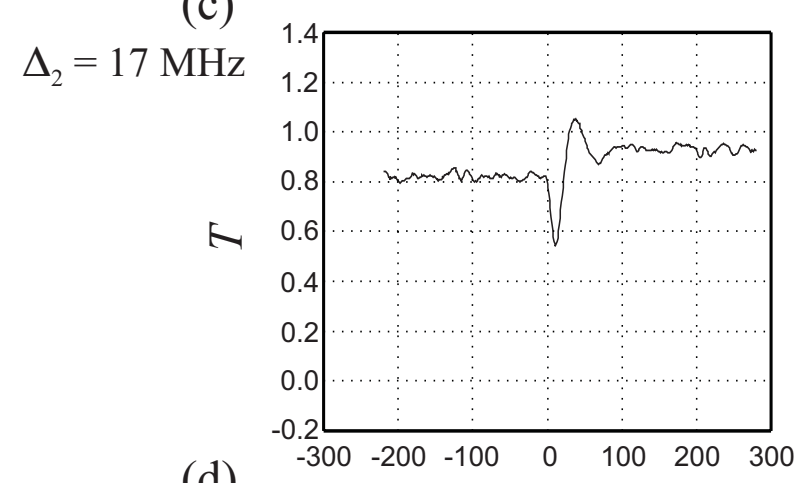

(d)

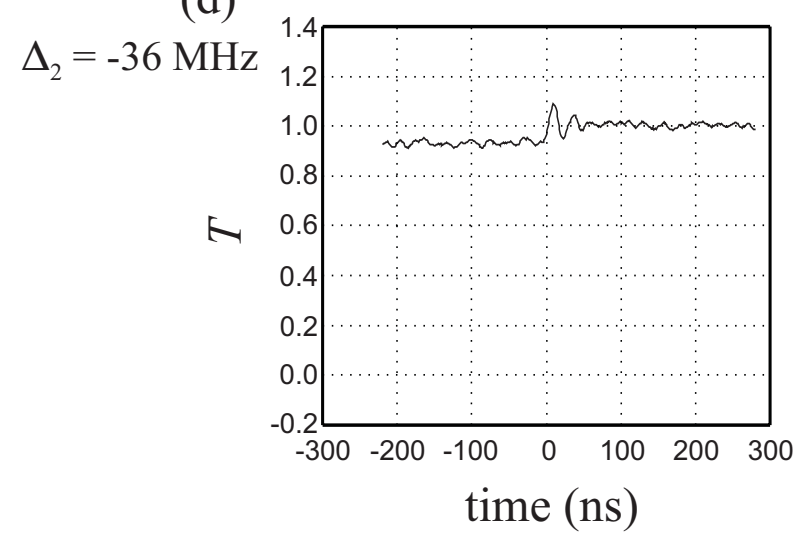

Theory
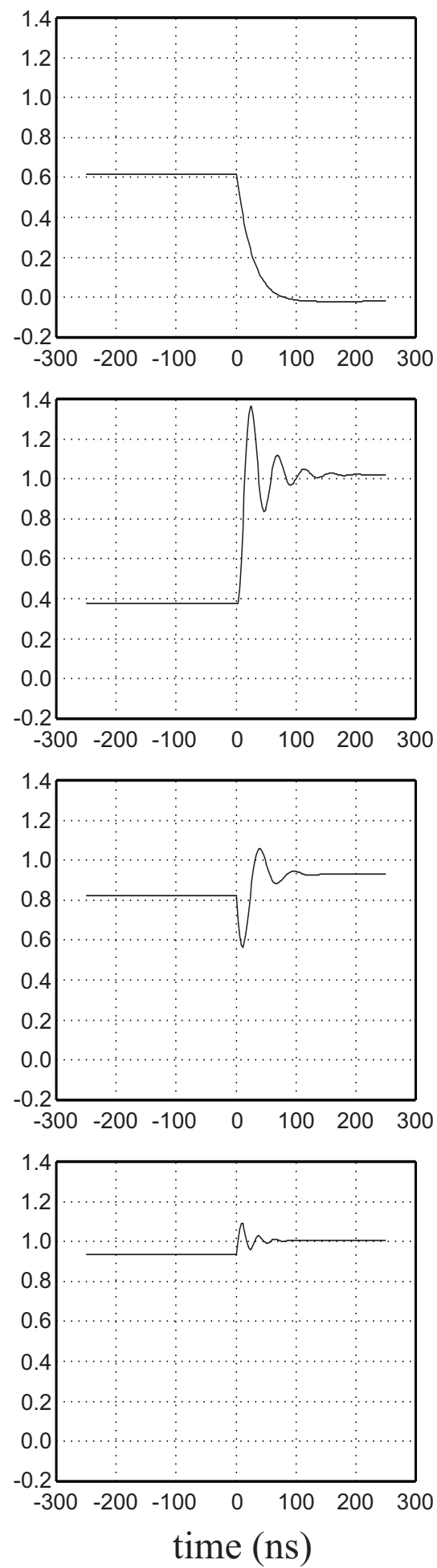

FIG. 7: Turn-off transients for a strong, on-resonant coupling field: (a) $\Delta_{2}=0 \mathrm{MHz}$ (bare state filling), (b) $\Delta_{2}=22 \mathrm{MHz}$ (dressed state emptying), (c) $\Delta_{2}=17 \mathrm{MHz}$ and (d) $\Delta_{2}=-36 \mathrm{MHz}$. In all cases $\Omega_{1}=46 \mathrm{MHz}$ before turn-off and the uncoupled absorption was found to be about $20 \%$. 
(a)

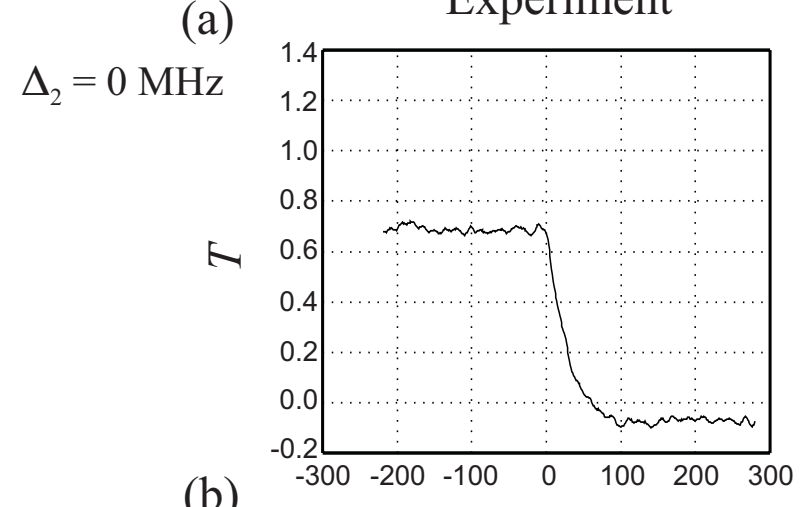

(b)

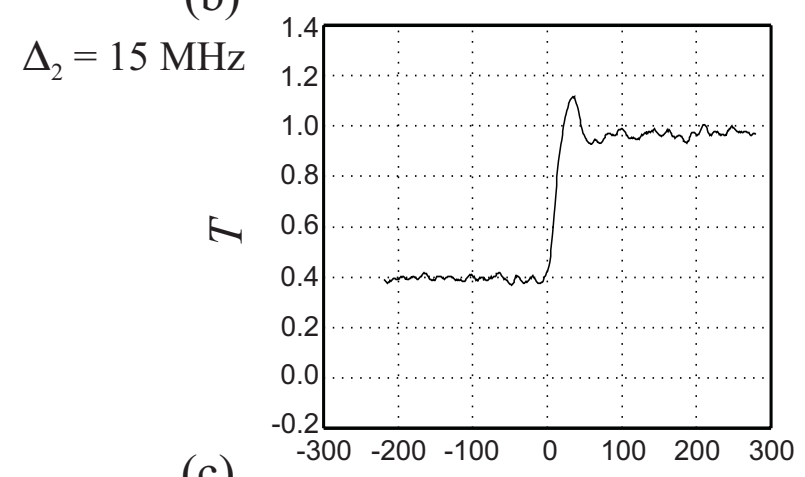

(c)

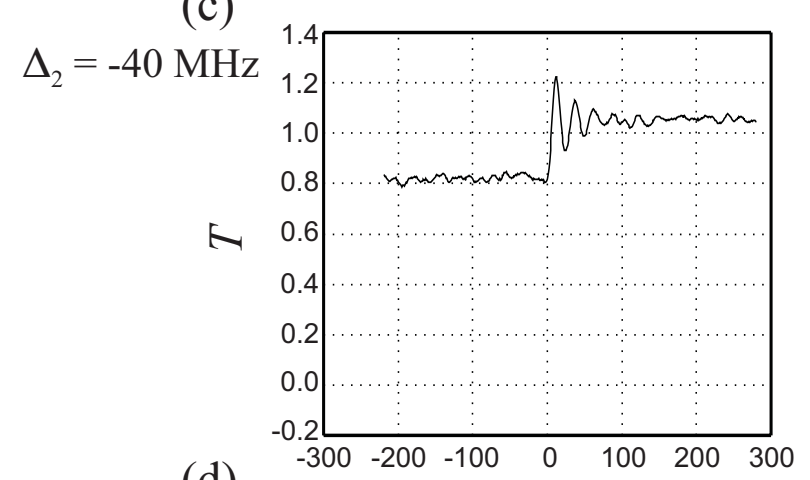

(d)

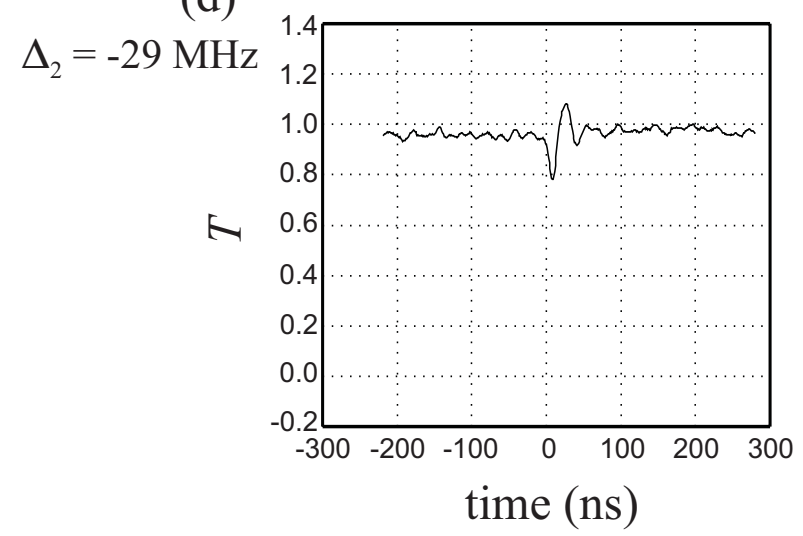

Theory
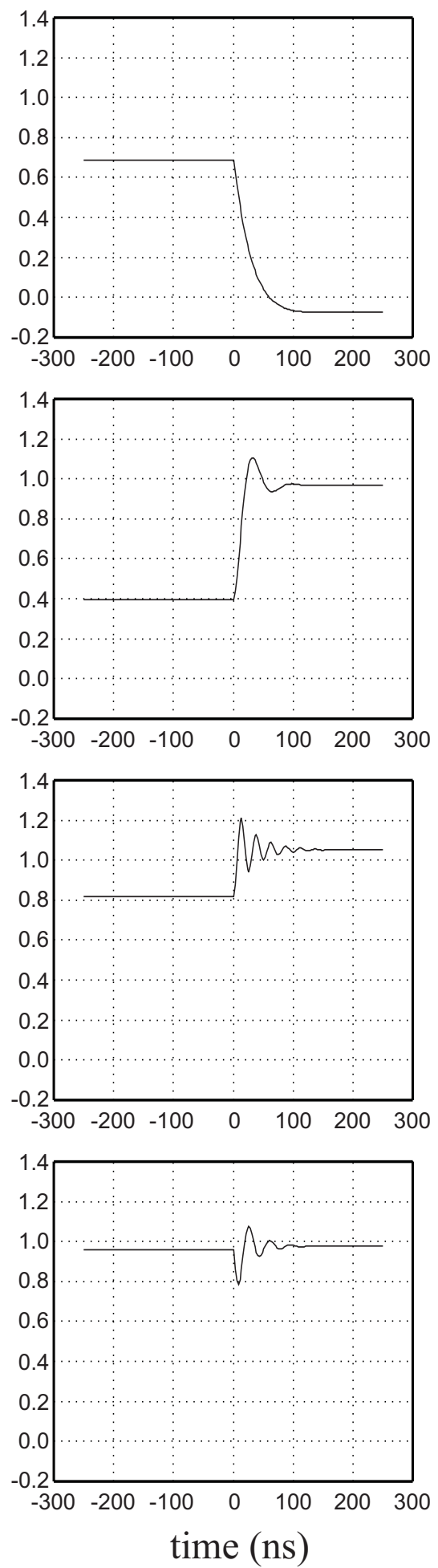

FIG. 8: Turn-off transients for a strong, off-resonant coupling field: (a) $\Delta_{2}=0 \mathrm{MHz}$ (bare state filling), (b) $\Delta_{2}=15 \mathrm{MHz}$ (major dressed state emptying), (c) $\Delta_{2}=-40 \mathrm{MHz}$ (minor dressed state emptying) and (d) $\Delta_{2}=-29 \mathrm{MHz}$. In all cases $\Delta_{1}=-23 \mathrm{MHz}, \Omega_{1}=46 \mathrm{MHz}$ before turn-off and the uncoupled absorption was found to be about $20 \%$. 


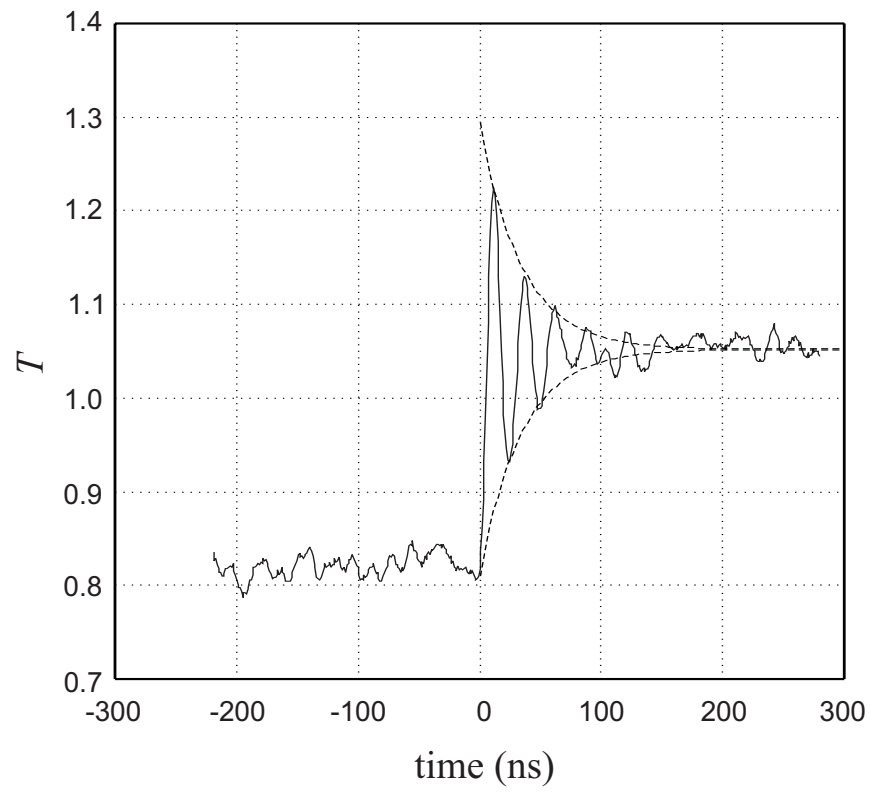

FIG. 9: Close-up of experimental FIG. 8(c) showing the minor dressed state emptying (solid curve) with added exponential decays overlayed (dashed curves). The inverse lifetime of the decay was found to be $5.5 \mathrm{MHz}$ which corresponds well with the measured probe spectral linewidths.

ported above. The very clear signal features a gain peak rising to $T=1.35$, making this another promising region for further study of transient gain. We note that this is a tunable gain region and is remote from any uncoupled absorptions. The figure also shows two full periods of oscillation. The measured period of oscillation was found to be $46 \mathrm{~ns}$ which is consistent with the probe detuning of $-22 \mathrm{MHz}$ determined from the cavity off-set voltage. Figures $\mathrm{Q}(\mathrm{c})$ and $(\mathrm{d})$ were taken for detunings of $\Delta_{2}=17 \mathrm{MHz}$ and $\Delta_{2}=-36 \mathrm{MHz}$ respectively. These results show that cycles of gain and absorption can be present even when there is little or no change in the steady state transmission.

The turn-off results for a detuned coupling field are shown in FIG. 8. Case (a) shows the transient filling of the bare state and is similar to FIG. E(a). Cases (b) and (c) show the major and minor dressed states emptying respectively. As in the case of a resonant coupling field, these transients are characterized by ringing and gain. In case (b) $\left(\Delta_{2}=15 \mathrm{MHz}\right.$, corresponding to the major dressed state) these effects are somewhat obscured by being in the wings of the uncoupled absorption. In contrast, case (c) $\left(\Delta_{2}=-40 \mathrm{MHz}\right.$, corresponding to the minor dressed state) shows larger gain and exceptionally clear ringing. A fit to the decay of the ringing is shown in FIG. 9. As expected, this gives a decay rate of $5.5 \mathrm{MHz}$, which corresponds to half the phenomenological decay rate measured from the probe spectral linewidths. Finally, FIG. $8(\mathrm{~d})$ is for a probe detuning of $\Delta_{2}=-29 \mathrm{MHz}$, again showing transient features between two regions of steady-state transparency.

\section{CONCLUSIONS}

We have carried out a theoretical and experimental study of transient phenomena associated with the switching of the coupling field in a $\Lambda$ type EIT system. These studies have shown novel transient gain features and aid the understanding of transient dressing.

We have experimentally observed interesting transient gain features in both the turn-on and turn-off regimes. We have found it useful to interpret the transient dynamics in terms of the filling and emptying of dressed states. The absolute peak gain values, especially the dressed state emptying, were found to be comparable in strength to the more usual, on-resonant turn-on case 23]. Off resonant gain peaks have an important advantage over resonant gain peaks in that they can be tuned by appropriate choice of coupling strengths and detunings. In this way they can, for example, be removed from uncoupled absorptions or perhaps used to match transitions of different frequencies. The turn-off experiments also exhibited extremely clear transient signals and as such may be useful as a tool to explore the properties of atomic systems.

We have obtained some new analytical results. In particular we give the weak probe response after turning off the coupling field for arbitrary detunings. Analysis of the resonant turn-on using the Laplace method enabled us to identify a frequency oscillation not reported previously. We analyzed this oscillation in terms of the 3D Vector Model and outlined a parameter regime where we would expect it to be observable.

These results have applicability to the understanding of EIT for quantum information storage and may lead the way to methods which avoid the limitations of the condition of adiabaticity inherent in present schemes for optical quantum information storage [7, 40].

\section{Acknowledgments}

The authors would like to acknowledge the financial support of the EPSRC, as well as technical support from Shahid Hanif (Imperial College, London), Roger Bence, Fraser Robertson and Robert Seaton (The Open University, Milton Keynes).

*

\section{APPENDIX A: FURTHER INVESTIGATIONS OF THE "FAST" OSCILLATIONS}

The oscillations in $\operatorname{Im}\left[\rho_{b c}(t)\right]$ at the angular frequency $\Omega_{1}$ noted in Section II are unusual in that the Rabi frequency usually associated with the cycling of population is approximately $\Omega_{1} / 2$. The origins of this effect in the absence of decay are easily demonstrated using the 3D Vector Model of 3-state systems introduced recently [34, 35]. 
The 3D Vector Model is based on the representation of the state of the atom $|\psi(t)\rangle=v_{a}|a\rangle+v_{b}|b\rangle-i v_{c}|c\rangle$ by the real, 3 -dimensional vector $\vec{v}(t)=\left(v_{a}, v_{b}, v_{c}\right)^{\mathrm{T}}$, where here the states $|a\rangle,|b\rangle$ and $|c\rangle$ represent the bare energy eigenstates of the atom. The components of $\vec{v}$ are given with respect to three orthogonal axes which we shall call the $a, b$ and $c$ axes. These axes represent the three energy eigenstates of the atom.

The action of the (resonant) probe and coupling fields on $|\psi\rangle$ is represented simply as a rotation about a $R a b i$ vector $\vec{\Omega}=\left(-\frac{1}{2} \Omega_{2}, \frac{1}{2} \Omega_{1}, 0\right)^{\mathrm{T}}$ as follows:

$$
\frac{d \vec{v}}{d t}=\vec{\Omega} \times \vec{v}
$$

The rotation of $\vec{v}$ about $\vec{\Omega}$ occurs at the frequency $\Omega \equiv$ $|\vec{\Omega}|=\left(\Omega_{1}^{2}+\Omega_{2}^{2}\right)^{1 / 2} / 2$ which is approximately $\Omega_{1} / 2$ for $\Omega_{2} \ll \Omega_{1}$. The Rabi vector lies in the $a-b$ plane at an angle of $\theta=\tan ^{-1}\left(\Omega_{2} / \Omega_{1}\right) \approx \Omega_{2} / \Omega_{1}$ to the $b$ axis. The value of $\rho_{b c}$ is the product of the amplitude of the state $|b\rangle$ and the complex conjugate of the amplitude of state $|c\rangle$ in $|\psi\rangle$, which corresponds to the product $i v_{b} v_{c}$, and so $\operatorname{Im}\left(\rho_{b c}\right)=v_{b} v_{c}$. Absorption of the probe occurs when $v_{b} v_{c}<0$ and gain when $v_{b} v_{c}>0$.

We note from Eq. (18) that, in general, Im $\left(\rho_{b c}\right)$ has frequency components at both the $\Omega_{1} / 2$ and $\Omega_{1}$ frequencies. However, there are two important cases where $\operatorname{Im}\left(\rho_{b c}\right)$ oscillates at either $\Omega_{1}$ or $\Omega_{1} / 2$.

The first case is when $\rho_{b b}^{0}=1$. Substituting this into Eq. 18) gives $\operatorname{Im}\left(\rho_{b c}\right)$ oscillating at a frequency of $\Omega_{1} / 2$. In this case the initial state vector lies along the $\pm b$ axis, that is, $\vec{v}(0)=(0, \pm 1,0)^{\mathrm{T}}$. In time the state vector $\vec{v}$ traces out an acute cone (of half angle $\theta$ ) centered on $\pm \vec{\Omega}$ and revolves at frequency $\Omega$. The full solution of Eq. (A1) is given by

$$
\vec{v}(t)= \pm\left(\begin{array}{c}
-\cos \theta \sin \theta[\cos (\Omega t)-1] \\
\cos ^{2} \theta+\sin ^{2} \theta \cos (\Omega t) \\
-\sin \theta \sin (\Omega t)
\end{array}\right) .
$$

Keeping terms up to first order in $\Omega_{2} / \Omega_{1}$ gives $\operatorname{Im}\left[\rho_{b c}(t)\right] \approx-\left(\Omega_{2} / \Omega_{1}\right) \sin (\Omega t) \approx-\left(\Omega_{2} / \Omega_{1}\right) \sin \left(\Omega_{1} t / 2\right)$ as found in Eq. (18).

The second case is when $\rho_{a a}^{0}=\rho_{b b}^{0}=1 / 2$. In this case Eq. (18) shows that $\operatorname{Im}\left(\rho_{b c}\right)$ oscillates only at the double frequency, i.e. $\Omega_{1}$. Equation (18) was derived for zero ground state coherence, i.e. $\rho_{a b}^{0}=0$. This means that in the gas sample, half the atoms are in state $|a\rangle$ and the remainder are in $|b\rangle$. We model this by taking the average of the values of $\operatorname{Im}\left(\rho_{b c}\right)$ for both collections of atoms. For the atoms beginning in the $|a\rangle$ state, the vector is initially aligned along the $\pm a$ axis, $\vec{v}(0)=( \pm 1,0,0)^{\mathrm{T}}$, and traces out a cone of half angle $\pi / 2 \pm \theta$. The solution of Eq. (A1) for this case is given by

$$
\vec{v}(t)= \pm\left(\begin{array}{c}
1+[\cos (\Omega t)-1] \cos ^{2} \theta \\
\cos \theta \sin \theta[\cos (\Omega t)-1] \\
-\cos \theta \sin (\Omega t)
\end{array}\right) .
$$

Keeping terms up to first order in $\Omega_{2} / \Omega_{1}$ gives $\operatorname{Im}\left[\rho_{b c}(t)\right] \approx-\left(\Omega_{2} / \Omega_{1}\right)\left[\frac{1}{2} \sin (2 \Omega t)-\sin (\Omega t)\right] . \quad$ Taking the average of this value and the value in Eq. A2 yields $\operatorname{Im}\left[\overline{\rho_{b c}(t)}\right] \approx-\left(\Omega_{2} / \Omega_{1}\right) \frac{1}{4} \sin (2 \Omega t) \approx$ $-\left(\Omega_{2} / \Omega_{1}\right) \frac{1}{4} \sin \left(\Omega_{1} t\right)$ as found in Eq. (18).

In summary, we have traced the origin of the oscillations at twice the Rabi frequency $\Omega_{1}$ to the fact that for a pure state, $\rho_{b c}$ is the product of the amplitude of finding the atom in state $|b\rangle$ and the complex conjugate of the amplitude in state $|c\rangle$. In the absence of decay, both amplitudes have terms that oscillate at the frequency $\Omega_{1} / 2$, and so their product gives rise to a term oscillating at twice this frequency. So even though the populations oscillate at the Rabi frequency $\Omega_{1} / 2$, the absorption and emission of light has a term which occurs at twice this frequency.
[1] S.E. Harris, J.E. Field and A. Imamoglu, Phys. Rev. Lett., 64, 1107-1110 (1990); G.Z. Zhang, K. Hakuta and B.P. Stoicheff, Phys. Rev. Lett 71, 3099 (1993); A. J. Merriam, S.J. Sharpe, H. Xia, D. Manuszak, G.Y. Yin and S.E. Harris, Opt. Lett. 24, 625 (1999); C. Dorman, I Kucukkara and J.P. Marangos, Phys. Rev. A, 61, 013802 (1999).

[2] C. F. Roos, D. Leibfried, A.Mundt, F. Schmidt-Kaler, J. Eschner and R. Blatt, Phys. Rev. Lett. 85, 5547, (2000); G. Morigi, J. Eschner, and C. H. Keitel, Phys. Rev. Lett. 85, 4458 (2000).

[3] K. M. Gheri, P. Grangier, J-P. Poizat and D. F. Walls, Phys. Rev. A 46, 4276-4285 (1992).

[4] J. Mompart and R. Corbalán, J. Opt. B: Quantum Semiclass. Opt. 2, R7 (2000).

[5] M. M. Kash, V. A. Sautenkov, A. S. Zibrov, L. Hollberg,
G. R. Welch, M. D. Lukin, Y. Rostovtsev, E. S. Fry and M. O. Scully, Phys. Rev. Lett., 82, 5229-5231 (1999); L. V. Hau, S. E. Harris, Z. Dutton and C. H. Behroozi, Nature, 397, 594-8, (1999); J. P. Marangos, ibid, 397, 559-560 (1999).

[6] A. M. Steinberg and R. Y. Chiao, Phys. Rev. A 49, 2071 (1994); L. J. Wang, A. Kuzmich, and A. Dogariu, Nature (London) 406, 277 (2000); A. Dogariu, A. Kuzmich, and L. J. Wang, Phys. Rev. A 63, 053806 (2001).

[7] O. Kocharovskaya, Y. Rostovtsev, M. O. Scully, Phys. Rev. Lett. 86, 628 (2001); C. Liu, Z. Dutton, C. H. Behroozi, and L. V. Hau, Nature (London) 409, 490 (2001); D. F. Phillips, A. Fleischhauer, A. Mair, and R. L. Walsworth, M. D. Lukin, Phys. Rev. Lett. 86, 783 (2001)

[8] A. Imamoglu, H. Schmidt, G. Woods and M. Deutsch, 
Phys. Rev. Lett., 79, 1467 (1997); S. E. Harris and L. V. Hau, Phys. Rev. Lett. 82, 4611 (1999); S. Rebić, S.M. Tan, A.S. Parkins and D.F. Walls, J. Opt. B: Quantum Semiclass. Opt. 1, 490 (1999); K.M. Gheri, W. Alge, and P. Grangier, Phys. Rev. A 60, R2673 (1999); A.D. Greentree, J.A. Vaccaro, S.R. de Echaniz, A.V. Durrant and J.P. Marangos, J.Opt. B: Quantum Semiclass. Opt 2 , 252-259 (2000).

[9] J. P . Marangos, J. Mod. Opt. 45, 471, (1998).

[10] P. R. Berman and R. Salomaa, Phys. Rev. A 25, 2667 (1982).

[11] N. Lu and P. R. Berman, Phys. Rev. A 36, 3845 (1987).

[12] N. Lu, P. R. Berman, A. G. Yodh, Y. S. Bai and T. W. Mossberg, Phys. Rev. A, 33, 3956 (1986).

[13] S. E. Harris and Z. F. Luo, Phys. Rev. A 52, R928 (1995).

[14] Y. Li and M. Xiao, Opt. Lett. 20, 1489-1491 (1995).

[15] Y. Zhu, Phys. Rev. A 53, 2742 (1996).

[16] Y. Zhu, Phys. Rev. A 55, 4568 (1997).

[17] Y. S. Bai, A. G. Yodh and T. W. Mossberg, Phys. Rev. Lett. 55, 1277 (1985).

[18] Y. S. Bai, T. W. Mossberg, N. Lu and P. R. Berman, Phys. Rev. Lett. 57, 1692 (1986).

[19] H. W. H. Lee and J. E. Wessel, Phys. Rev. Lett. 59, 1416 (1987).

[20] E. S. Fry, X. Li, D. Nikonov, G. G. Padmabandu, M. O. Scully, A. V. Smith, F. K. Tittel, C. Wang, S. R. Wilkinson, and S-Y. Zhu, Phys. Rev. Lett. 70, 3235, (1993).

[21] C. Wei, A. D. Greentree, N. B. Manson and J. P. D. Martin, J. Lumin. 66 \& 67, 61 (1996); C. Wei, S. A. Holmstrom, A. D. Greentree and N. B. Manson, J. Opt. B: Quantum Semiclass. Opt. 1, 289 (1999).

[22] H. X. Chen, A. V. Durrant, J. P. Marangos and J. A. Vaccaro, Phys. Rev. A 58, 1545 (1998)

[23] S. R. de Echaniz, A. D. Greentree, A. V. Durrant, D. M. Segal, J. P. Marangos, J. A. Vaccaro, Observation of transient gain without population inversion in a laser-cooled rubidium lambda system, quant-ph/0105084, accepted for publication in Phys. Rev. A
[24] A. Schenzle and R. G. Brewer, Phys. Rev. A 14, 1756 (1976).

[25] S. E. Harris and Y. Yamamoto, Phys. Rev. Lett. 81, 3611 (1998).

[26] M. Yan, E. G. Rickey and Y. Zhu, Observation of absorptive photon switching by quantum interference, preprint, accepted for publication in Phys. Rev. A.

[27] for a recent review see N. V. Vitanov, T. Halfmann, B. W. Shore and K. Bergmann, Annu. Rev. Phys. Chem. 52, 763 (2001).

[28] A. Nottelmann, C. Peters, and W. Lange, Phys. Rev. Lett., 70, 1783 (1993).

[29] O. D. Mücke, T. Tritschler, M. Wegener, U. Morgner and F. X. Kärtner, Phys. Rev. Lett. 87, 057401 (2001)

[30] S.A. Holmstrom, A.S.M. Windsor, C. Wei, J.P.D. Martin and N.B. Manson, Journal of Luminescence $\mathbf{7 6}$ \& 77, 38 (1998).

[31] P. M. Morse and H. Feshbach, Methods of theoretical physics, (McGraw-Hill, New York 1953).

[32] P.L. Knight and P.W. Milonni, Physics Reports-Review Section of Physics Letters 66, 2, 21 (1980).

[33] Mathematica 4.1, Wolfram Research Inc., (2000).

[34] A. Kasapi, J. Opt. Soc. Am. B. 13, 1347 (1996).

[35] J.A. Vaccaro, A.V. Durrant, S.A. Hopkins, H. Chen, K.E. Hill, J. Mod. Opt. 45, 315 (1998).

[36] S. R. de Echaniz, A. D. Greentree, A. V. Durrant, D. M. Segal, J. P. Marangos, J. A. Vaccaro, Phys. Rev. A 64, 013812 (2001).

[37] S. H. Autler and C. H. Townes, Phys. Rev. 100, 703 (1955).

[38] D. McGloin, M.H. Dunn, and D.J. Fulton, Phys. Rev. A 62, 053802 (2000).

[39] Origin 6.0284, Microcal Software Inc., (2000).

[40] We note, however, that there are other schemes for realising fast quantum gate operations, see for example D. Jaksch, J. I. Cirac, P. Zoller, S. L. Rolston, R. Côté and M. D. Lukin, Phys. Rev. Lett. 85, 2208 (2000). 